Supporting Information

\title{
A Novel Synthesis of Functionalized Allylsilane
}

\author{
Wei-Dong Z. Li* and Jin-Hui Yang
}

State Key Laboratory of Applied Organic Chemistry, Lanzhou University, Lanzhou 730000, China

\section{Experimental}

\section{General}

For product purification by flash column chromatography, silica gel (200 300 mesh) and light petroleum ether (bp. $30 \sim 60{ }^{\circ} \mathrm{C}$ or $60 \sim 90{ }^{\circ} \mathrm{C}$ ) are used. All solvents were purified and dried by standard techniques, and distilled prior to use. All organic extracts were dried over $\mathrm{MgSO}_{4}$ or $\mathrm{Na}_{2} \mathrm{SO}_{4}$, unless otherwise noted. IR spectra were recorded on a Nicolet FT-170 SX spectrometer as liquid film. ${ }^{1} \mathrm{H}$ and ${ }^{13} \mathrm{C} \mathrm{NMR}$ spectra were recorded on a Bruker AM-200, AM-400or Mercury PLUS-300 spectrometer with TMS as an internal standard and $\mathrm{CDCl}_{3}$ as solvent. HRMS were determined on a Bruker Daltonics APEXII 47e FT-ICR spectrometer.

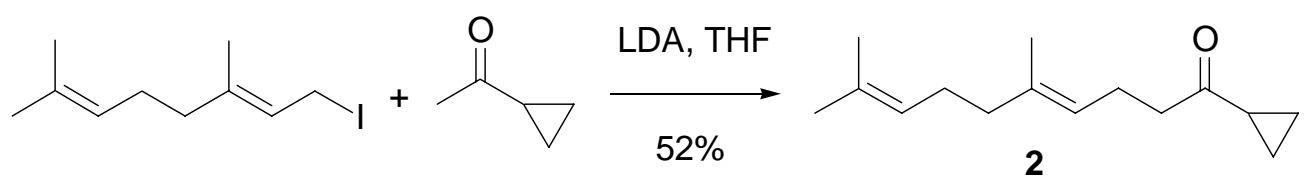

To a stirred solution of diisopropylamine $(0.6 \mathrm{~mL}, 4.2 \mathrm{mmol})$ in dry THF $(6 \mathrm{~mL})$ was added $n$-BuLi $(1.6 \mathrm{M}, 3.6 \mathrm{mmol})$ under $\mathrm{N}_{2}$ at $0{ }^{\circ} \mathrm{C}$. After being stirred for $15 \mathrm{~min}$., the rection mixture was cooled to $-78^{\circ} \mathrm{C}$, and then the solution of cyclopropyl methyl ketone $(252 \mathrm{mg}, 3 \mathrm{mmol})$ in THF $(2 \mathrm{~mL})$ was added dropwise via syringe. After being stirred for $30 \mathrm{~min}$. at $-78{ }^{\circ} \mathrm{C}$, the solution of geranyl iodide ${ }^{1}(672 \mathrm{mg}, 2.5 \mathrm{mmol})$ in THF $(2 \mathrm{~mL})$ was added into the reaction mixture. Stirring was continuted until the temperature of the reaction mixture was warmed gradually to room temperature. The solution was diluted with $\mathrm{Et}_{2} \mathrm{O}$, and washed with saturated aqueous $\mathrm{NH}_{4} \mathrm{Cl}$, water and brine. The extract was dried over anhydrous $\mathrm{Na}_{2} \mathrm{SO}_{4}$ and followed by removal of the solvent in vacuo to give a residue, which after purification by flash column chromatography on silica gel (1/32 EtOAc-Petro. ether) afforded 2 (293 mg, 52\%) as a colorless oil. IR $(\mathrm{KBr}) v_{\max } 1699,1384,1081 \mathrm{~cm}^{-1} ;{ }^{1} \mathrm{H} \mathrm{NMR}\left(\mathrm{CDCl}_{3}, 200 \mathrm{MHz}\right): \delta$ 5.09(2H, m, CH), 2.57(2H, t, $\left.J=8 \mathrm{~Hz}, \mathrm{CH}_{2}\right), 2.29\left(2 \mathrm{H}, \mathrm{m}, \mathrm{CH}_{2}\right), 2.07-1.86(7 \mathrm{H}, \mathrm{m}, \mathrm{CH}$, $\left.3 \mathrm{CH}_{2}\right), 1.68\left(\mathrm{~s}, \mathrm{CH}_{3}\right), 1.61\left(\mathrm{~s}, \mathrm{CH}_{3}\right), 1.60\left(\mathrm{~s}, \mathrm{CH}_{3}\right), 0.99\left(2 \mathrm{H}, \mathrm{m}, \mathrm{CH}_{2}\right), 0.85\left(2 \mathrm{H}, \mathrm{m}, \mathrm{CH}_{2}\right)$

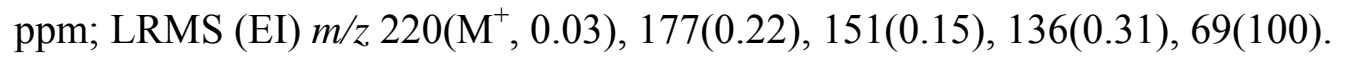

\footnotetext{
${ }^{1}$ Freshly prepared from geraniol by a standard method $\left(\mathrm{I}_{2}, \mathrm{Ph}_{3} \mathrm{P}\right.$, imidazole, $\mathrm{CH}_{3} \mathrm{CN}-\mathrm{Et}_{2} \mathrm{O}$, r.t.).
} 


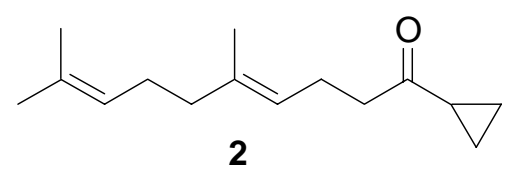

2

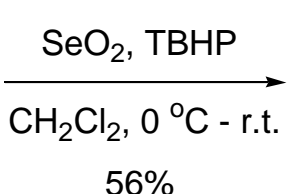

$56 \%$

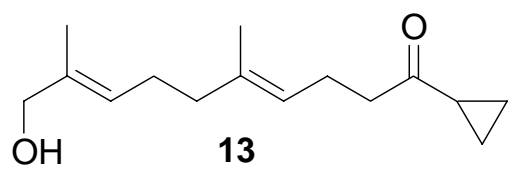

13

To a stirred solution of $\mathrm{SeO}_{2}(523 \mathrm{mg}, 4.7 \mathrm{mmol})$ and tert-butyl hydroperoxide $(70 \%, 6.06 \mathrm{~g}, 47.1 \mathrm{mmol})$ in $50 \mathrm{~mL}$ of $\mathrm{CH}_{2} \mathrm{Cl}_{2}$ was added compound $2(5.8 \mathrm{~g}, 26.5$ $\mathrm{mmol}$ ) at $0{ }^{\circ} \mathrm{C}$ over $20 \mathrm{~min}$. Stirring was continuted for $2 \mathrm{~h}$. The resulting mixture was diluted with ether, then washed with $10 \%$ aqueous $\mathrm{KOH}$, water and brine, dried, and concentrated to give a yellow liquid residue, which after purification by flash column chromatography on silica gel (1/4 EtOAc-Petro. ether) afforded 13 (3.50 g, 56\%) as a colorless oil, and recovered starting material 2 (1.20 g). 13, IR (KBr) $v_{\max } 3425,1695$, 1388, $1012 \mathrm{~cm}^{-1}$; ${ }^{1} \mathrm{H}$ NMR $\left(\mathrm{CDCl}_{3}, 300 \mathrm{MHz}\right): \delta 5.34(1 \mathrm{H}, \mathrm{t}, J=6.8 \mathrm{~Hz}, \mathrm{CH})$, $5.08(1 \mathrm{H}, \mathrm{t}, J=7 \mathrm{~Hz}, \mathrm{CH}), 3.98\left(2 \mathrm{H}, \mathrm{d}, J=4.5 \mathrm{~Hz}, \mathrm{CH}_{2} \mathrm{O}\right), 2.61\left(2 \mathrm{H}, \mathrm{m}, \mathrm{CH}_{2}\right), 2.33(2 \mathrm{H}$, $\left.\mathrm{m}, \mathrm{CH}_{2}\right), 2.09-1.93\left(5 \mathrm{H}, \mathrm{m}, \mathrm{CH}, 2 \mathrm{CH}_{2}\right), 1.72\left(3 \mathrm{H}, \mathrm{s}, \mathrm{CH}_{3}\right), 1.65\left(3 \mathrm{H}, \mathrm{s}, \mathrm{CH}_{3}\right), 0.99(2 \mathrm{H}$, $\left.\mathrm{m}, \mathrm{CH}_{2}\right), 0.87\left(2 \mathrm{H}, \mathrm{m}, \mathrm{CH}_{2}\right) \mathrm{ppm}$; LRMS (EI) $\mathrm{m} / z 236\left(\mathrm{M}^{+}, 0.03\right), 218(0.12), 167$ (1.5), 69 (100).

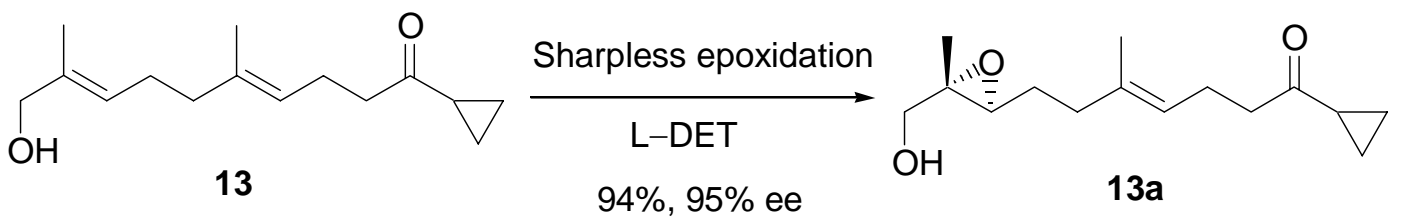

To a mixture of $\operatorname{Ti}\left(\mathrm{O}^{i} \mathrm{Pr}\right)_{4}(4.0 \mathrm{~mL}, 13.47 \mathrm{mmol}), \mathrm{CaH}_{2}(32 \mathrm{mg}, 1.35 \mathrm{mmol})$, silica gel $(50 \mathrm{mg})$ and molecular sieves $(50 \mathrm{mg})$ in $20 \mathrm{~mL}$ of dry $\mathrm{CH}_{2} \mathrm{Cl}_{2}$ was injected L-(+)-diethyl tartrate (DET) $(3.33 \mathrm{~g}, 16.16 \mathrm{mmol})$ via syringe under $\mathrm{N}_{2}$ at $0{ }^{\circ} \mathrm{C}$. After 10 minutes the solution of $13(3.18 \mathrm{~g}, 13.47 \mathrm{mmol})$ in $5 \mathrm{~mL}$ of dry $\mathrm{CH}_{2} \mathrm{Cl}_{2}$ was injected. The reaction mixture was stirred for another $10 \mathrm{~min}$. The anhydrous TBHP (2.8 $\mathrm{M}$ in toluene, $26.88 \mathrm{mmol}$ ) of $9.6 \mathrm{~mL}$ was then injected at $-40{ }^{\circ} \mathrm{C}$ under $\mathrm{N}_{2}$. The resulting mixture was stirred another $4 \mathrm{~h}$ on keeping the temperature of system to lower $-20{ }^{\circ} \mathrm{C}$. TLC showed that a small amount of starting material remained. The aqueous tartaric solution of $35 \mathrm{~mL}(10 \%)$ was added at $-20^{\circ} \mathrm{C}$. Stirring was continued at room temp. until the aqueous layer became clear. The reaction mixture was extracted with diethyl ether and washed with $5 \%$ aqueous $\mathrm{NaOH}$, saturated aqueous $\mathrm{NaHCO}_{3}$, water and brine, dried over anhydrous $\mathrm{Na}_{2} \mathrm{SO}_{4}$. Removal of the solvent in vacuo followed by flash column chromatography on silica gel (1/2 EtOAc-Petro. ether) afforded epoxide $13 \mathbf{a}\left(3.20 \mathrm{~g}, 94 \%, 95 \%\right.$ ee) as a colorless oil. $[\alpha]_{\mathrm{D}}{ }^{25}-9.1$ (c 4.4, $\left.\mathrm{CHCl}_{3}\right)$; IR (KBr) $v_{\max } 3447,1695,1388,1084,1039 \mathrm{~cm}^{-1} ;{ }^{1} \mathrm{H} \mathrm{NMR}\left(\mathrm{CDCl}_{3}, 300\right.$ $\mathrm{MHz}): \delta 5.15(1 \mathrm{H}, \mathrm{t}, J=6.8 \mathrm{~Hz}, \mathrm{CH}), 3.62\left(2 \mathrm{H}, \mathrm{dd}, J_{l}=12 \mathrm{~Hz}, J_{2}=26.2 \mathrm{~Hz}, \mathrm{OCH}_{2}\right)$, $3.00\left(1 \mathrm{H}, \mathrm{t}, J=6.2 \mathrm{~Hz}\right.$, epoxy-H), $2.57\left(2 \mathrm{H}, \mathrm{t}, J=7.2 \mathrm{~Hz}, \mathrm{CH}_{2}\right), 2.33\left(2 \mathrm{H}, \mathrm{m}, \mathrm{CH}_{2}\right)$, 2.10(2H, m, $\left.\mathrm{CH}_{2}\right), 1.94(1 \mathrm{H}, \mathrm{m}, \mathrm{CH}), 1.72\left(2 \mathrm{H}, \mathrm{m}, \mathrm{CH}_{2}\right), 1.67\left(\mathrm{~s}, \mathrm{CH}_{3}\right), 1.27\left(\mathrm{~s}, \mathrm{CH}_{3}\right)$, $1.02\left(2 \mathrm{H}, \mathrm{m}, \mathrm{CH}_{2}\right), 0.86\left(2 \mathrm{H}, \mathrm{m}, \mathrm{CH}_{2}\right) \mathrm{ppm}$; HRMS (ESI) calcd for $\left(\mathrm{M}+\mathrm{NH}_{4}\right)^{+}$, 270.2640, found for $\left(\mathrm{M}+\mathrm{NH}_{4}\right)^{+}: 270.2640$. 


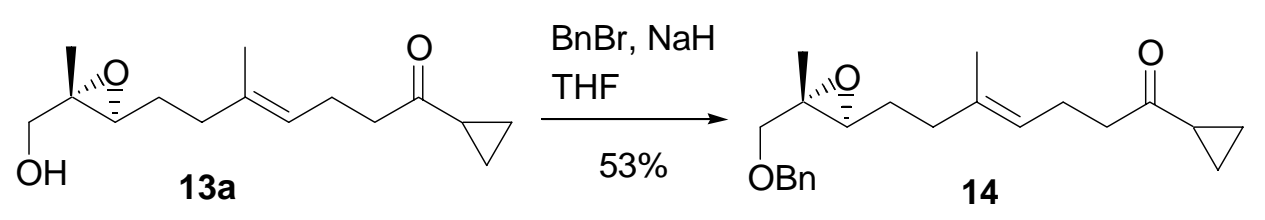

A solution epoxide 13a $(3.80 \mathrm{~g}, 15.1 \mathrm{mmol})$ in $5 \mathrm{~mL}$ THF was added to the a suspension of sodium hydride (60\% in mineral oil, $0.72 \mathrm{~g}, 18.0 \mathrm{mmol}$ ) in $20 \mathrm{~mL}$ of dry THF and the mixture was stirred for $10 \mathrm{~min}$. under $\mathrm{N}_{2}$. The solution of benzyl bromide (3.36 g, $19.6 \mathrm{mmol})$ and $n$-tetrabutylammonium iodide $(279 \mathrm{mg}, 0.76 \mathrm{mmol})$ in $10 \mathrm{~mL}$ THF was added and the mixture was stirred for $2 \mathrm{~h}$. Saturated aqueous $\mathrm{NH}_{4} \mathrm{Cl}(20 \mathrm{~mL})$ was added the mixture and extracted with diethyl ether. The combined organic layers were washed with water and brine, dried and the solvent removed under reduced pressure. Column chromatography of the residue (1/8 EtOAc-Petro. ether) gave the title compound $\mathbf{1 4}(1.59 \mathrm{~g}, 53 \%)$ as a colorless oil, and the starting epoxide 13a $(1.576 \mathrm{~g})$ was recovered. 14, $[\alpha]_{\mathrm{D}}{ }^{25}-3.6\left(c 3.7, \mathrm{CHCl}_{3}\right)$; IR $(\mathrm{KBr}) v_{\max }$ 1697, 1386, $1090 \mathrm{~cm}^{-1} ;{ }^{1} \mathrm{H} \mathrm{NMR}\left(\mathrm{CDCl}_{3}, 300 \mathrm{MHz}\right): \delta 7.26(5 \mathrm{H}, \mathrm{m}, \mathrm{Ar}-\mathrm{H}), 5.07(1 \mathrm{H}, \mathrm{t}$, $J=6.9 \mathrm{~Hz}, \mathrm{CH}), 4.48\left(2 \mathrm{H}, \mathrm{d}, J=4.2 \mathrm{~Hz}, \mathrm{CH}_{2}\right), 3.39\left(2 \mathrm{H}, \mathrm{dd}, J_{1}=11.2 \mathrm{~Hz}, J_{2}=24.2\right.$ $\left.\mathrm{Hz}, \mathrm{OCH}_{2}\right), 2.77\left(1 \mathrm{H}, \mathrm{t}, J=6.3 \mathrm{~Hz}\right.$, epoxy-H), $2.49\left(2 \mathrm{H}, \mathrm{t}, J=7.2 \mathrm{~Hz}, \mathrm{CH}_{2}\right), 2.21(2 \mathrm{H}$, $\left.\mathrm{m}, \mathrm{CH}_{2}\right), 2 . .07\left(2 \mathrm{H}, \mathrm{m}, \mathrm{CH}_{2}\right), 1.81(1 \mathrm{H}, \mathrm{m}, \mathrm{CH}), 1.60\left(2 \mathrm{H}, \mathrm{m}, \mathrm{CH}_{2}\right), 1.58\left(3 \mathrm{H}, \mathrm{s}, \mathrm{CH}_{3}\right)$, $1.25\left(3 \mathrm{H}, \mathrm{s}, \mathrm{CH}_{3}\right), 0.92\left(2 \mathrm{H}, \mathrm{m}, \mathrm{CH}_{2}\right), 0.77\left(2 \mathrm{H}, \mathrm{m}, \mathrm{CH}_{2}\right) \mathrm{ppm}$; HRMS (ESI) calcd for $\left(\mathrm{M}+\mathrm{NH}_{4}\right)^{+}, 360.2533$, found for $\left(\mathrm{M}+\mathrm{NH}_{4}\right)^{+}: 360.2541$.

\section{General procedure for the preparation of allysilane from cyclopropyl ketone}

An oven-dried, three-necked $50 \mathrm{~mL}$ round bottomed flask, fitted with a nitrogen line, rubber septum, vacuum line and stirrer bar, was thoroughly flashed with dry nitrogen and charged with cerium (III) chloride heptahydrate (296 mg, $1.2 \mathrm{mmol}$ ). The flask was heated under vacuum $(1 \mathrm{mmHg})$ at $150^{\circ} \mathrm{C}$ for $7 \mathrm{~h}$ with vigorous stirring, resulting in a white mobile solid. The reaction flask was flashed with nitrogen and allowed to cool to $20^{\circ} \mathrm{C}$ when dry THF $(10 \mathrm{ml})$ was added to the vigorously stirred cerium (III) chloride to form a uniform white suspension, which was left with vigorous stirring for $2 \mathrm{~h}$.

Preparation of (dimethylphenylsilyl)methyl magnesium chloride: A three-necked $25 \mathrm{~mL}$ flask fitted with a condenser, pressure equalised dropping funnel, a rubber septum and a stirrer bar was charged with magnesium turnings (10 g) and the whole apparatus was flame dried under a flow of nitrogen. Chloromethyldimethylphenyl silane $(12 \mathrm{mmol})$ in dry $\mathrm{Et}_{2} \mathrm{O}(12 \mathrm{ml})$ was added dropwise under an atmosphere of nitrogen to form a gray solution $(\sim 1 \mathrm{M})$, which was stirred for $1 \mathrm{~h}$ until all the magnesium metal was consumed.

The dry cerium (III) chloride suspension was cooled to $0{ }^{\circ} \mathrm{C}$ with stirring, to which the Grignard reagent $(1.2 \mathrm{~mL}, 1.2 \mathrm{mmol})$ prepared above was added dropwise to give a suspension. After stirring for $1 \mathrm{~h}$ at $0{ }^{\circ} \mathrm{C}$, cyclopropyl ketone $(1 \mathrm{mmol})$ in 1 $\mathrm{mL}$ of THF was added dropwise and the resulting mixture was warmed gradually to room temperature, whilst the reaction was monitored by TLC. When consumption of 
starting ketone was complete (by TLC), the reaction mixture was cooled to $0{ }^{\circ} \mathrm{C}$ and quenched by the dropwise addition of water $(1 \mathrm{~mL})$, the organic layer was separated and the aqueous phase was extracted with diethyl ether. The organic layers were washed with water, brine and dried $\left(\mathrm{MgSO}_{4}\right)$. The solvent was evaporated in vacuo at $0{ }^{\circ} \mathrm{C}$ carefully to give a yellow liquid residue, which was taken in anhydrous diethyl ether $(10 \mathrm{~mL})$ under nitrogen atmosphere and a freshly prepared $\mathrm{MgI}_{2} \cdot\left(\mathrm{OEt}_{2}\right)_{\mathrm{n}}(1.1$ mmol, $0.25 \mathrm{M})$ solution ${ }^{2}$ in $\mathrm{Et}_{2} \mathrm{O}$-benzene $(1: 1)$ was added dropwise. The resulting mixture was stirred for $10 \mathrm{~min}$. at $0{ }^{\circ} \mathrm{C}$ and quenched with satd. $\mathrm{NaHCO}_{3}$, extracted with diethyl ether. The organic layer was washed with $10 \%$ sodiun thiosulfate solution, water, brine and dried $\left(\mathrm{MgSO}_{4}\right)$. The solvent was evaporated in vacuo to give a pale yellow oily residue, which was purified by flash chromatography using diethyl ether-petro. ether (bp. 30 60 ${ }^{\circ} \mathrm{C}$ ) as eluent to afford the allysilane as a colorless oil.

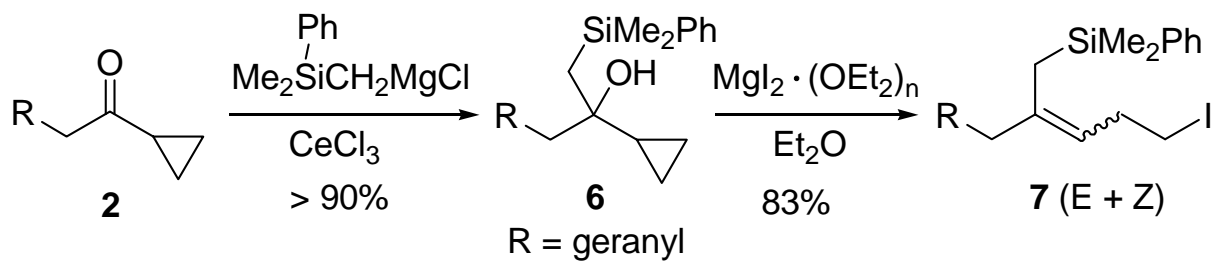

Allylsilane 7 was prepared from cyclopropyl ketone $\mathbf{2}$ as an inseparable (by chromatographic silica gel) mixture of geometric isomers $(78 \%, E / Z 1: 1)$. IR ( $\mathrm{KBr})$ $v_{\max } 3390,2927,1728,1650,1614,1579 \mathrm{~cm}^{-1} ;{ }^{1} \mathrm{H} \mathrm{NMR}\left(\mathrm{CDCl}_{3}, 300 \mathrm{MHz}\right): \delta$ 7.50-7.40(5H, m, Ar-H), 5.06(1H, m, CH), 4.97(1H, t, $J=6.4 \mathrm{~Hz}, \mathrm{CH}(2-Z)), 4.88(1 \mathrm{H}$, $\mathrm{t}, J=6.1 \mathrm{~Hz}, \mathrm{CH}(2-E)), 3.02\left(2 \mathrm{H}, \mathrm{t}, J=7.5 \mathrm{~Hz}, \mathrm{CH}_{2} \mathrm{I}\right), 2.94\left(2 \mathrm{H}, \mathrm{t}, J=7.5 \mathrm{~Hz}, \mathrm{CH}_{2} \mathrm{I}\right)$, $2.57\left(2 \mathrm{H}, \mathrm{dd}, J_{1}=7.5 \mathrm{~Hz}, J_{2}=14.6 \mathrm{~Hz}, \mathrm{CH}_{2}\right), 2.34\left(2 \mathrm{H}, \mathrm{dd}, J_{1}=7.5 \mathrm{~Hz}, J_{2}=14.4 \mathrm{~Hz}\right.$, $\left.\mathrm{CH}_{2}\right), 2.13-1.70\left(8 \mathrm{H}, \mathrm{m}, 4 \mathrm{CH}_{2}\right), 1.72\left(2 \mathrm{H}, \mathrm{m}, \mathrm{CH}_{2}\right), 1.68\left(\mathrm{~s}, \mathrm{CH}_{3}\right), 1.60\left(\mathrm{~s}, \mathrm{CH}_{3}\right), 1.56(\mathrm{~s}$, $\left.\mathrm{CH}_{3}\right), 0.31\left(6 \mathrm{H}, \mathrm{s}, 2 \mathrm{CH}_{3}\right) \mathrm{ppm} ;{ }^{13} \mathrm{C} \mathrm{NMR}\left(\mathrm{CDCl}_{3}, 75 \mathrm{MHz}\right): \delta 139.49,139.09,135.52$, $135.14,133.61,133.49,131.28,129.08,128.93,127.81,127.69,124.36,123.92$, 123.66, 122.01, 120.84, 39.68, 38.95, 32.83, 32.49, 32.42, 26.69, 26.02, 25.68, 21.26, 17.69, 16.02, 6.50(C-I), 6.02(C-I), -2.34, -2.72(C-Si) ppm; LRMS (EI) m/z $480\left(\mathrm{M}^{+}\right.$, 1.2), 353 (0.5), 135 (100); HRMS (EI) calcd for $(\mathrm{M})^{+}, 480.1704$, found for $(\mathrm{M})^{+}$: 480.1702 .

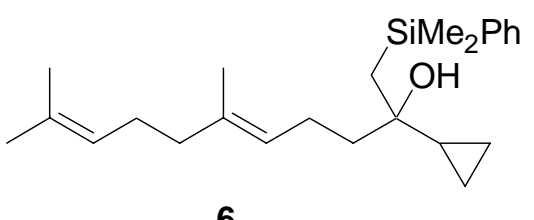

6 (> 90\%), colorless oil. IR (KBr) $v_{\max } 3477,1429,1249,1112,835 \mathrm{~cm}^{-1} ;{ }^{1} \mathrm{H}$ NMR $\left(\mathrm{CDCl}_{3}, 200 \mathrm{MHz}\right): \delta$ 7.55-7.34(5H, m, Ar-H), 5.04(2H, m, CH), 2.17-1.99(6H, $\left.\mathrm{m}, 3 \mathrm{CH}_{2}\right), 1.68\left(\mathrm{~s}, \mathrm{CH}_{3}\right), 1.60\left(\mathrm{~s}, \mathrm{CH}_{3}\right), 1.58\left(\mathrm{~s}, \mathrm{CH}_{3}\right), 1.24\left(2 \mathrm{H}, \mathrm{m}, \mathrm{CH}_{2}\right), 1.16(1 \mathrm{H}, \mathrm{m}$, $\mathrm{CH}), 0.88\left(4 \mathrm{H}, \mathrm{m}, \mathrm{CH}_{2}\right), 0.38\left(6 \mathrm{H}, \mathrm{s}, 2 \mathrm{CH}_{3}\right), 0.34\left(2 \mathrm{H}, \mathrm{m}, \mathrm{CH}_{2}\right) \mathrm{ppm}$; LRMS (EI) $\mathrm{m} / \mathrm{z}$ $308\left(\mathrm{M}^{+}, 0.01\right), 290(0.07), 247(0.2), 220(0.28), 73$ (100).

\footnotetext{
${ }^{2}$ Li, W. -D. Z.; Zhang, X. -X. Org. Lett. 2002, 4, 3485.
} 


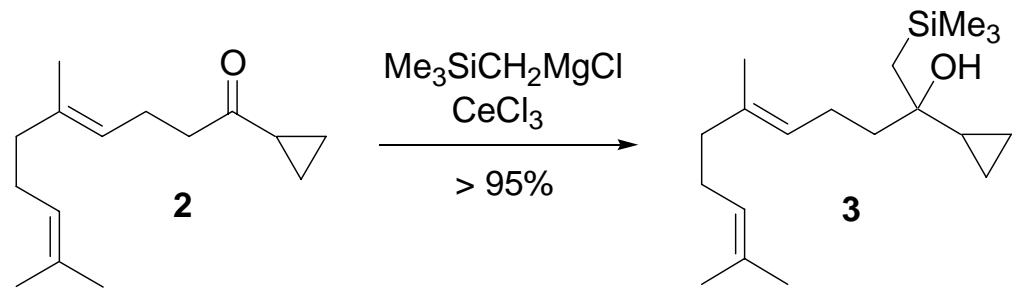

Cyclopropyl carbinol 3 was prepared analogously as for $\mathbf{6}$ from the (trimethylsilyl)methyl cerium reagent ${ }^{3}$ and ketone $\mathbf{2}$ in $95 \%$ yield, as a colorless oil. IR (KBr) $v_{\max } 3417,1247,1021,839 \mathrm{~cm}^{-1} ;{ }^{1} \mathrm{H} \mathrm{NMR}\left(\mathrm{CDCl}_{3}, 200 \mathrm{MHz}\right): \delta 5.36(2 \mathrm{H}$, $\mathrm{m}, 2 \mathrm{CH}), 2.40-2.23\left(6 \mathrm{H}, \mathrm{m}, 3 \mathrm{CH}_{2}\right), 1.90,1.84,1.83\left(9, \mathrm{~s}, 3 \mathrm{CH}_{3}\right), 1.77\left(2 \mathrm{H}, \mathrm{m}, \mathrm{CH}_{2}\right)$, $1.20\left(2 \mathrm{H}, \mathrm{s}, \mathrm{SiCH}_{2}\right), 1.10(1 \mathrm{H}, \mathrm{m}, \mathrm{CH}), 0.57\left(4 \mathrm{H}, \mathrm{m}, 2 \mathrm{CH}_{2}\right), 0.30\left(9 \mathrm{H}, \mathrm{s}, 3 \mathrm{CH}_{3}\right) \mathrm{ppm}$; LRMS (EI) $\mathrm{m} / 2,290$ ( $\left.\mathrm{M}^{+}-18,0.18\right), 247$ (0.1), 157 (1.2), 73 (100).<smiles>C=C(C)C1CC(=O)[C@]2(C)C[C@H]2C1</smiles><smiles>CCOCCOCCOCC</smiles><smiles>C=C(C)[C@H]1CC(C[AsH2])=C(C)[C@H](CI)C1</smiles>

Allylsilane 9 was prepared from ketone $\mathbf{8}^{4}$ as a colorless oil $(68 \%)$. $[\alpha]_{\mathrm{D}}{ }^{25}+5.5$ (c 7.4, $\left.\mathrm{CHCl}_{3}\right)$; IR (KBr) $v_{\max } 1645,1251,1114,832 \mathrm{~cm}^{-1} ;{ }^{1} \mathrm{H}$ NMR $\left(\mathrm{CDCl}_{3}, 300\right.$ MHz): $\delta$ 7.48-7.35(5H, m, Ar-H), 4.69(1H, s, CH), 4.64(1H, s, CH), 3.40(1H, d, $J=$ $\left.10.2 \mathrm{~Hz}, \mathrm{CH}_{2} \mathrm{I}\right), 2.97\left(2 \mathrm{H}, \mathrm{t}, J=10.5 \mathrm{~Hz}, \mathrm{CH}_{2} \mathrm{I}\right), 2.33(1 \mathrm{H}, \mathrm{m}, \mathrm{CH}), 2.04(2 \mathrm{H}, \mathrm{d}, J=11.4$ $\left.\mathrm{Hz}, \mathrm{CH}_{2}\right), 1.81(1 \mathrm{H}, \mathrm{d}, J=13.5 \mathrm{~Hz}, \mathrm{CH}), 1.69\left(2 \mathrm{H}, \mathrm{m}, \mathrm{CH}_{2}\right), 1.65\left(3 \mathrm{H}, \mathrm{s}, \mathrm{CH}_{3}\right)$, $1.56\left(2 \mathrm{H}, \mathrm{m}, \mathrm{CH}_{2}\right), 1.53\left(\mathrm{~s}, \mathrm{CH}_{3}\right), 0.30\left(6 \mathrm{H}, \mathrm{s}, 2 \mathrm{CH}_{3}\right) \mathrm{ppm} ;{ }^{13} \mathrm{C} \mathrm{NMR}\left(\mathrm{CDCl}_{3}, 75 \mathrm{MHz}\right)$ : $\delta$ 149.1, 133.5, 131.1, 129.0, 127.7, 123.5, 108.7, 44.4, 38.3, 36.0, 31.9, 23.9, 20.8,

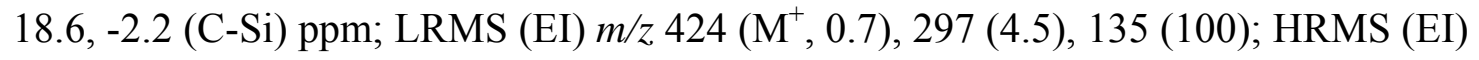
calcd for $(\mathrm{M})^{+}, 424.1078$, found for $(\mathrm{M})^{+}: 424.1068$.

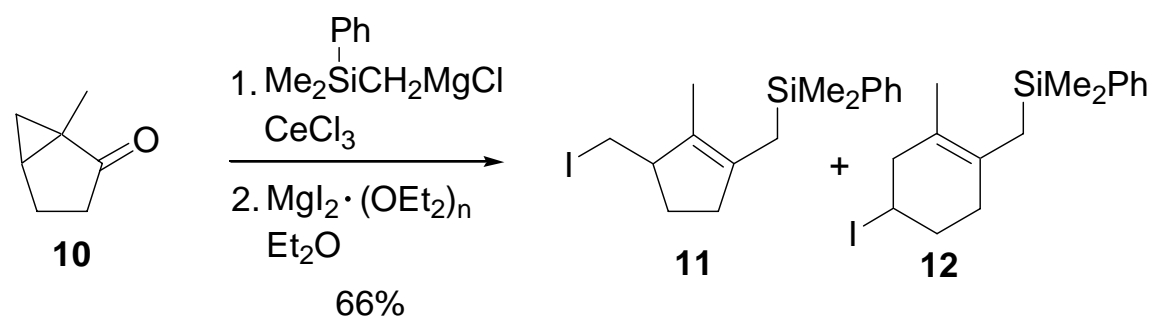

Allylsilanes $\mathbf{1 1}$ and $\mathbf{1 2}$ were prepared from ketone $\mathbf{1 0}^{4}$ as a mixture of $1.2: 1$ as a colorless oil (66\%). The ratio was determined by compare integral of $\mathrm{H} @ 3.36$ ppm with that of $\mathrm{H} @ 4.42$ ppm. IR (KBr) $v_{\max } 1428,1254,1060,831 \mathrm{~cm}^{-1}$; ${ }^{1} \mathrm{H}$ NMR

\footnotetext{
3 Prepared analogously as above from (chloromethyl)trimethylsilane.

${ }^{4}$ Prepared from the corresponding enones according to: (a) Corey, E. J.; Chaykovsky, M. J. Am. Chem. Soc. 1965, 87, 1353. (b) Chandrasekhar, S.; Narasihmulu, C.; Jagadeshwar, V.; Reddy, K. V. Tetrahedron Lett. 2003, 44, 3629.
} 
$\left(\mathrm{CDCl}_{3}, 300 \mathrm{MHz}\right): \delta$ 7.56-7.38(5H, m, Ar-H), 4.42(1H, m, CH-I(Compound 12)), $3.36\left(1 \mathrm{H}, \mathrm{dd}, J_{l}=2.8 \mathrm{~Hz}, J_{2}=9.4 \mathrm{~Hz}, \mathrm{CH}_{2} \mathrm{I}\right.$ (Compound 11)), $3.11\left(1 \mathrm{H}, \mathrm{dd}, J_{l}=7.2 \mathrm{~Hz}\right.$, $J_{2}=9.6 \mathrm{~Hz}, \mathrm{CH}_{2} \mathrm{I}$ (Compound 11)), 2.62(1H, m, CH), 2.18-1.46(5H, m, CH, 2CH $)$, 1.43(s, $\left.\mathrm{CH}_{3}\right), 0.32\left(\mathrm{~s}, \mathrm{CH}_{3}\right), 0.31\left(\mathrm{~s}, \mathrm{CH}_{3}\right) \mathrm{ppm} ;{ }^{13} \mathrm{C} \mathrm{NMR}\left(\mathrm{CDCl}_{3}, 75 \mathrm{MHz}\right): \delta 140.1$, 139.4, 135.9, 133.5, 133.0, 129.6, 129.2, 128.9, 127.7, 126.3, 122.2, 51.3, 44.3, 36.4, 35.6, 32.7, 29.4, 28.6, 23.4, 19.4, 19.1, 16.1, 12.2, -2.1, -2.1 (C-Si) ppm; LRMS (EI) $\mathrm{m} / \mathrm{z} 370\left(\mathrm{M}^{+}, 4.8\right), 243(4.2), 135$ (100); HRMS (EI) calcd for $(\mathrm{M})^{+}, 370.0608$, found for $(\mathrm{M})^{+}: 370.0606$.

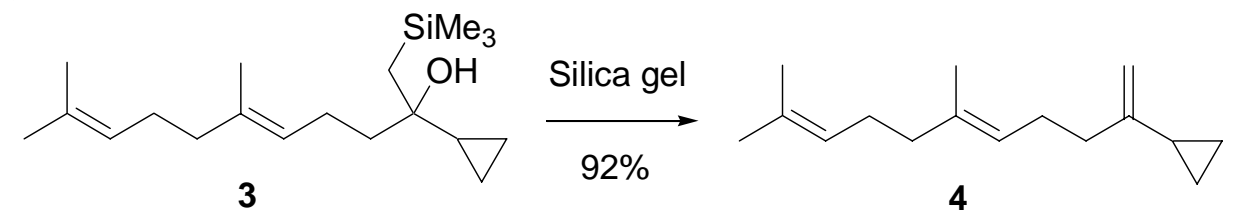

The alcohol 3 (308 mg, $1 \mathrm{mmol}$ ) was dissolved in $\mathrm{CH}_{2} \mathrm{Cl}_{2}(5 \mathrm{~mL})$ and silica gel (200-300 mesh, ca. 2 g) was added. The mixture was stirred at room temperature until TLC indicated the complete reaction when the silica gel was filtered off and thoroughly washed with dichloromethane $(2 \times 10 \mathrm{ml})$. The solvent was removed to give a residue, which after purification by flash column chromatography on silica gel (petrol ether) afforded cyclopropyl alkene 4 (204 mg, 92\%) as a colorless oil. IR (KBr) $v_{\max } 2924,1641,1446,880 \mathrm{~cm}^{-1} ;{ }^{1} \mathrm{H} \mathrm{NMR}\left(\mathrm{CDCl}_{3}, 200 \mathrm{MHz}\right): \delta 5.12(2 \mathrm{H}, \mathrm{m}, 2 \mathrm{CH})$, 4.62(2H, s, $\left.\mathrm{CH}_{2}\right), 2.20-2.00\left(8 \mathrm{H}, \mathrm{m}, 4 \mathrm{CH}_{2}\right), 1.67\left(\mathrm{~s}, \mathrm{CH}_{3}\right), 1.60\left(\mathrm{~s}, \mathrm{CH}_{3}\right), 1.30(1 \mathrm{H}, \mathrm{m}$, $\mathrm{CH}), 0.62\left(2 \mathrm{H}, \mathrm{m}, \mathrm{CH}_{2}\right), 0.45\left(2 \mathrm{H}, \mathrm{m}, \mathrm{CH}_{2}\right) \mathrm{ppm}$; LRMS (EI) m/z $218\left(\mathrm{M}^{+}, 0.3\right), 203$ (0.4), 147 (4.6), 69 (81), 41 (100).<smiles>CC1=C(CCC(=O)C2CC2)[C@]2(COc3ccccc3)OC2CC1</smiles>

14<smiles>CC1=C(CCC(=CCCI)CSC2(Cc3ccccc3)OC2Cc2ccccc2)CO1</smiles>

$17(\mathrm{E} / \mathrm{Z} 1: 1.8)$

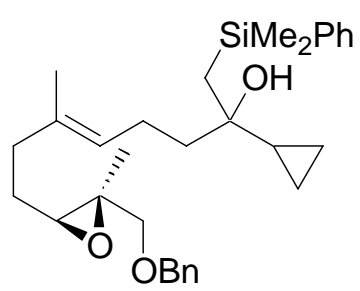

15<smiles>C1CC1</smiles>

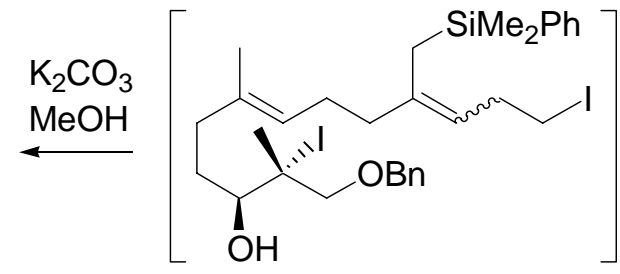

16

Compound 17 was prepared from ketone 14 as a colorless oil, via intermediate 16, which was transformed into compound 17 (58\% from 14) by the brief treatment with $\mathrm{K}_{2} \mathrm{CO}_{3}$ in methanol at r.t. for 0.5 h. 17, $[\alpha]_{\mathrm{D}}{ }^{25}+3\left(c 1.5, \mathrm{CHCl}_{3}\right)$; IR $(\mathrm{KBr}) v_{\max }$ 1451, 1249, 1109, $832 \mathrm{~cm}^{-1} ;{ }^{1} \mathrm{H}$ NMR $\left(\mathrm{CDCl}_{3}, 300 \mathrm{MHz}\right): \delta 7.49-7.33(5 \mathrm{H}, \mathrm{m}, \mathrm{Ar}-\mathrm{H})$, 5.07(1H, m, CH), 4.93(1H, t, $J=6.4 \mathrm{~Hz}, \mathrm{CH}(2 \mathrm{Z})), 4.88(1 \mathrm{H}, \mathrm{t}, J=6.1 \mathrm{~Hz}, \mathrm{CH}(2 E))$, $4.55\left(2 \mathrm{H}, \mathrm{d}, J=4.8 \mathrm{~Hz}, \mathrm{CH}_{2}\right), 3.47\left(2 \mathrm{H}, \mathrm{dd}, J_{1}=11.4 \mathrm{~Hz}, J_{2}=24.2 \mathrm{~Hz}, \mathrm{CH}_{2} \mathrm{O}\right)$, $3.02\left(2 \mathrm{H}, \mathrm{t}, J=7.3 \mathrm{~Hz}, \mathrm{CH}_{2} \mathrm{I}\right), 2.95\left(2 \mathrm{H}, \mathrm{t}, J=7.6 \mathrm{~Hz}, \mathrm{CH}_{2} \mathrm{I}\right), 2.83(1 \mathrm{H}, \mathrm{t}, J=6.2 \mathrm{~Hz}$, 
epoxy-H), 2.53(2H, dd, $\left.J_{1}=7.8 \mathrm{~Hz}, J_{2}=14.7 \mathrm{~Hz}, \mathrm{CH}_{2}\right), 2.36\left(2 \mathrm{H}, \mathrm{dd}, J_{l}=7.2 \mathrm{~Hz}, J_{2}\right.$ $\left.=14.7 \mathrm{~Hz}, \mathrm{CH}_{2}\right), 2.15-1.97\left(4 \mathrm{H}, \mathrm{m}, 2 \mathrm{CH}_{2}\right), 1.86\left(2 \mathrm{H}, \mathrm{m}, \mathrm{CH}_{2}\right), 1.73-1.57(4 \mathrm{H}, \mathrm{m}$,

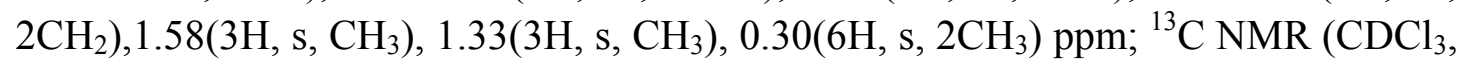
$75 \mathrm{MHz}): \delta 139.3,138.9,138.1,134.4,134.1,133.6,133.5,133.0,129.1,128.4$, 127.6, 124.9, 124.6, 124.4, 122.2, 120.9, 109.7, 106.1, 73.1, 72.2, 60.6, 59.8, 39.7, $38.8,36.2,35.9,32.7,32.5,32.2,26.8,26.7,26.0,21.2,16.0,14.5,-2.4,-2.8(\mathrm{C}-\mathrm{Si})$ ppm; HRMS (ESI) calcd for $\left(\mathrm{M}+\mathrm{NH}_{4}\right)^{+}, 620.2415$, found for $\left(\mathrm{M}+\mathrm{NH}_{4}\right)^{+}: 620.2425$.

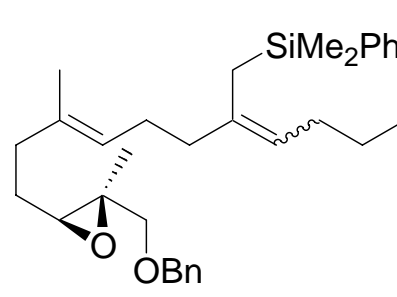

$17(\mathrm{E} / \mathrm{Z} 1: 1.8)$

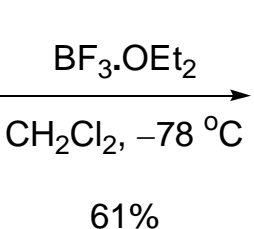

$61 \%$

To a stirred solution of 7 (30 mg, $0.05 \mathrm{mmol})$ in $20 \mathrm{~mL}$ dry $\mathrm{CH}_{2} \mathrm{Cl}_{2}$ was added $\mathrm{BF}_{3} \cdot \mathrm{Et}_{2} \mathrm{O}(0.025 \mathrm{~mL}, 0.25 \mathrm{mmol})$ under $\mathrm{N}_{2}$ at $-78^{\circ} \mathrm{C}$. After the solution was stirred at $-78{ }^{\circ} \mathrm{C}$ for 15 minutes, the reaction mixture was quenched at $-78{ }^{\circ} \mathrm{C}$ by adding saturated aqueous $\mathrm{NaHCO}_{3}(1 \mathrm{~mL})$. The resulting solution was allowed to warm to room temperature. The aqueous was extracted with diethyl ether. The combined organic extracts were dried $\left(\mathrm{MgSO}_{4}\right)$ and concentrated under reduced pressure. Isolation of the bicyclic products by flash chromatography on silica gel (1/8 EtOAc-Petro.) gave a mixture of the two compounds (ca. 1:1, $14 \mathrm{mg}, 61 \%$ ), which were separated by HPLC (40/1 hexane-isopropanol) to afford compound $\mathbf{1 8}$ as a colorless oil, and compound 19 as a colorless oil. Compound 18: $[\alpha]_{\mathrm{D}}{ }^{20}-52$ (c 0.4, $\left.\mathrm{CHCl}_{3}\right)$; IR (KBr) $v_{\max } 3451,1451,1089 \mathrm{~cm}^{-1} ;{ }^{1} \mathrm{H} \mathrm{NMR}\left(\mathrm{CDCl}_{3}, 300 \mathrm{MHz}\right): \delta$ 7.34 $(5 \mathrm{H}, \mathrm{m}, \mathrm{Ar}-\mathrm{H}), 4.77(1 \mathrm{H}$, brs, $\mathrm{CH}), 4.68(1 \mathrm{H}, \mathrm{brs}, \mathrm{CH}), 4.52(2 \mathrm{H}, \mathrm{d}, J=12 \mathrm{~Hz}$, $\left.\mathrm{OCH}_{2}\right), 3.60(1 \mathrm{H}, \mathrm{m}, \mathrm{OCH}), 3.49(1 \mathrm{H}, \mathrm{d}, J=8.4 \mathrm{~Hz}, \mathrm{OCH}), 3.23(1 \mathrm{H}, \mathrm{d}, J=8.4 \mathrm{~Hz}$, $\mathrm{OCH}), 3.17(1 \mathrm{H}, \mathrm{m}, \mathrm{ICH}), 2.86\left(1 \mathrm{H}, \mathrm{dd}, J_{1}=8.2 \mathrm{~Hz}, J_{2}=16.9 \mathrm{~Hz}, \mathrm{ICH}\right)$, 2.18-1.12(12H, m, 2CH, 5CH $), 1.25\left(3 \mathrm{H}, \mathrm{s}, \mathrm{CH}_{3}\right), 0.96\left(\mathrm{~s}, \mathrm{CH}_{3}\right), 0.89\left(\mathrm{~s}, \mathrm{CH}_{3}\right) \mathrm{ppm}$; ${ }^{13} \mathrm{C} \mathrm{NMR}\left(\mathrm{CDCl}_{3}, 75 \mathrm{MHz}\right): \delta 146.3,137.9,128.5,127.8,127.5,111.3,80.0,73.6$, 58.0, 42.0, 40.6, 37.5, 33.9, 30.7, 30.4, 29.7, 26.4, 23.3, 22.5 ppm; LRMS (EI) $\mathrm{m} / \mathrm{z}$ $468\left(\mathrm{M}^{+}, 1.3\right), 450(0.7), 341$ (2.1), 329 (7.1), 91 (100); HRMS (ESI) calcd for (M+H) ${ }^{+}, 469.1598$, found for $(\mathrm{M}+\mathrm{H})^{+}: 469.1601$.

Compound 19: $[\alpha]_{\mathrm{D}}{ }^{20} 19\left(c 0.2 \mathrm{CHCl}_{3}\right)$; IR (KBr) $v_{\max } 3448,1449,1091 \mathrm{~cm}^{-1}$; ${ }^{1} \mathrm{H}$ NMR $\left(\mathrm{CDCl}_{3}, 300 \mathrm{MHz}\right): \delta 7.32(5 \mathrm{H}, \mathrm{m}, \mathrm{Ar}-\mathrm{H}), 4.84(1 \mathrm{H}$, brs, $\mathrm{CH}), 4.54(1 \mathrm{H}, \mathrm{o}$, $\mathrm{CH}), 4.49\left(2 \mathrm{H}, \mathrm{o}, \mathrm{OCH}_{2}\right), 3.68(1 \mathrm{H}, \mathrm{m}, \mathrm{OCH}), 3.49(1 \mathrm{H}, \mathrm{d}, J=8.4 \mathrm{~Hz}, \mathrm{OCH}), 3.32(1 \mathrm{H}$, $\mathrm{m}, \mathrm{ICH}), 3.23(1 \mathrm{H}, \mathrm{d}, J=9 \mathrm{~Hz}, \mathrm{OCH}), 3.04\left(1 \mathrm{H}, \mathrm{d}, J_{l}=7.5 \mathrm{~Hz}, \mathrm{ICH}\right), 2.38-1.12(12 \mathrm{H}$, $\left.\mathrm{m}, 2 \mathrm{CH}, 5 \mathrm{CH}_{2}\right), 1.31\left(3 \mathrm{H}, \mathrm{s}, \mathrm{CH}_{3}\right), 0.87\left(\mathrm{~s}, \mathrm{CH}_{3}\right), 0.71\left(\mathrm{~s}, \mathrm{CH}_{3}\right) \mathrm{ppm} ;{ }^{13} \mathrm{C} \mathrm{NMR}\left(\mathrm{CDCl}_{3}\right.$, $75 \mathrm{MHz}): \delta 146.7,137.9,131.9,128.5,127.6,127.5,106.7,77.4,73.6,57.2,49.3$, 42.2, 39.2, 37.6, 36.4, 29.7, 28.8, 26.6, 22.5, $15.1 \mathrm{ppm}$; LRMS (EI) $\mathrm{m} / z .688\left(\mathrm{M}^{+}, 1.3\right)$, 450 (0.7), 341 (2.1), 329 (7.1), 91 (100); HRMS (ESI) calcd for (M+H) ${ }^{+}, 469.1598$, found for $(\mathrm{M}+\mathrm{H})^{+}: 469.1601$. 


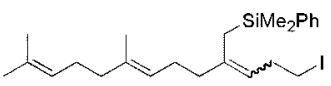

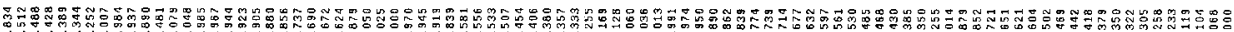

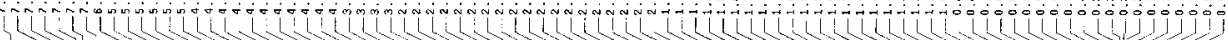
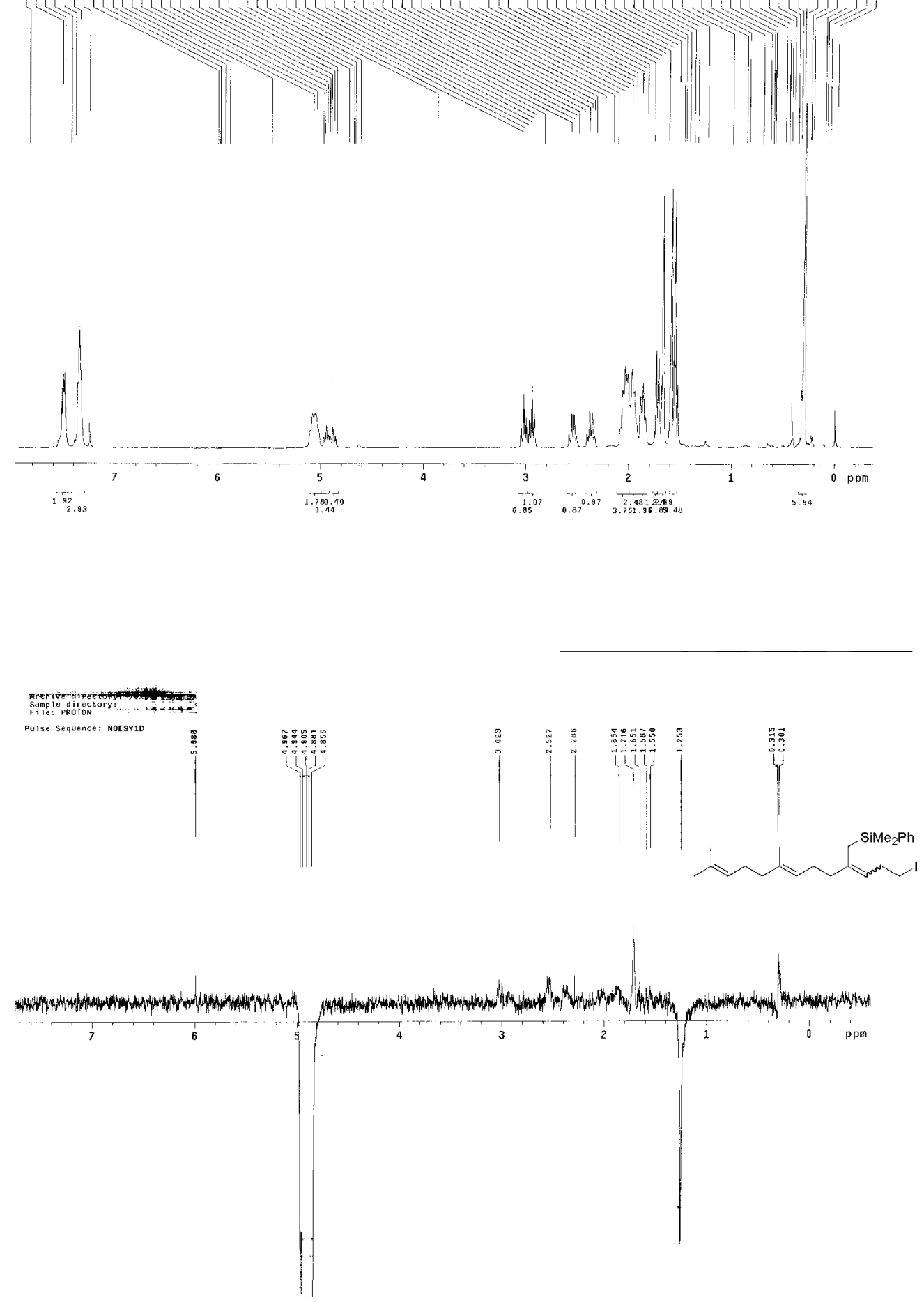


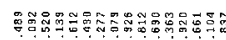

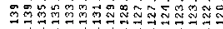

(1)

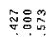

[i]

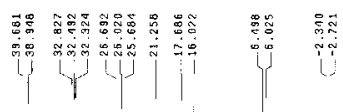

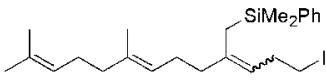

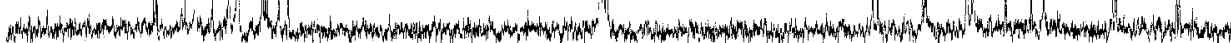
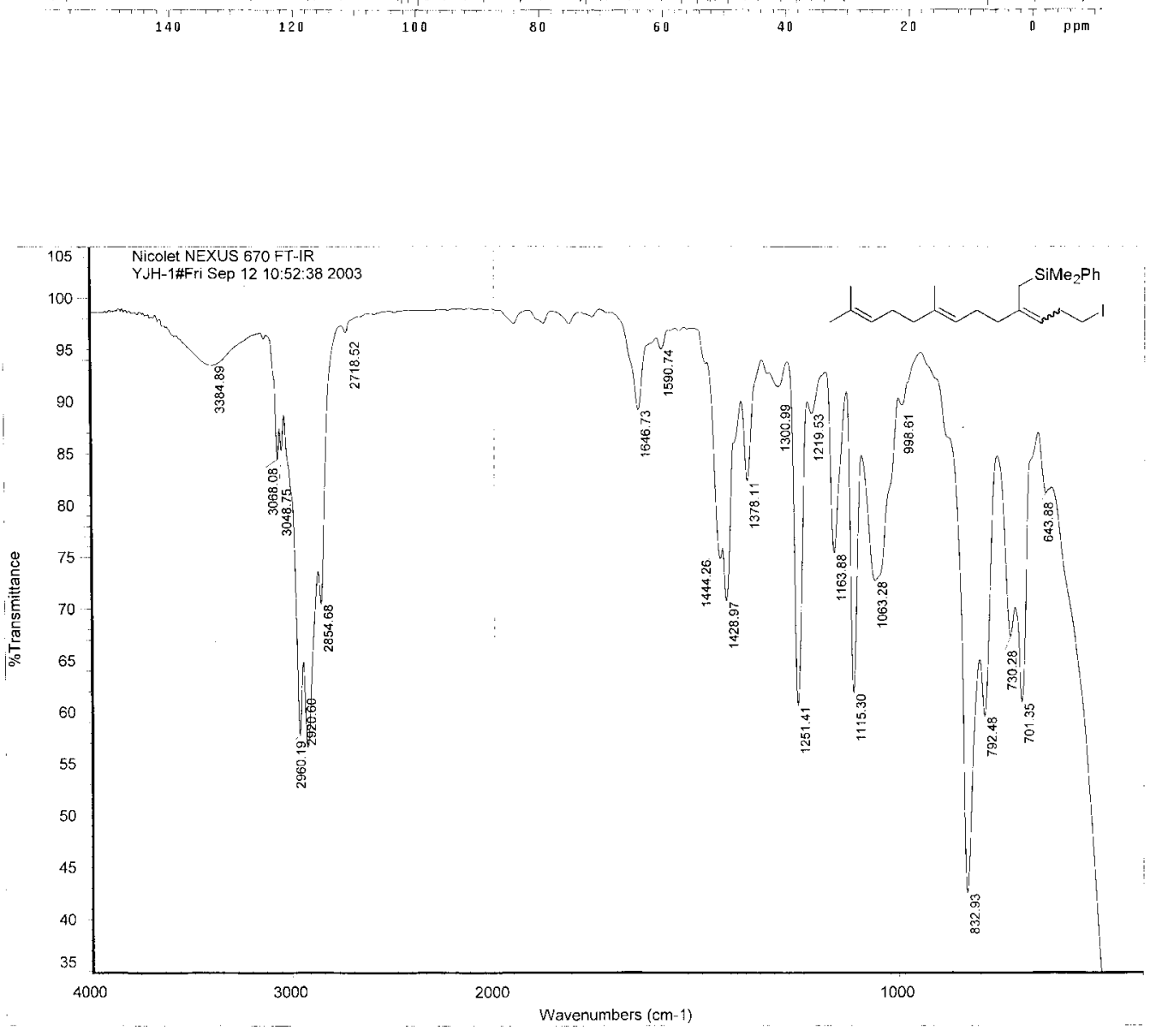
$\mathrm{yjh}-2$

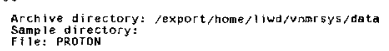

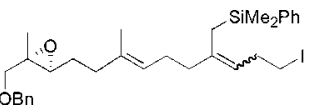
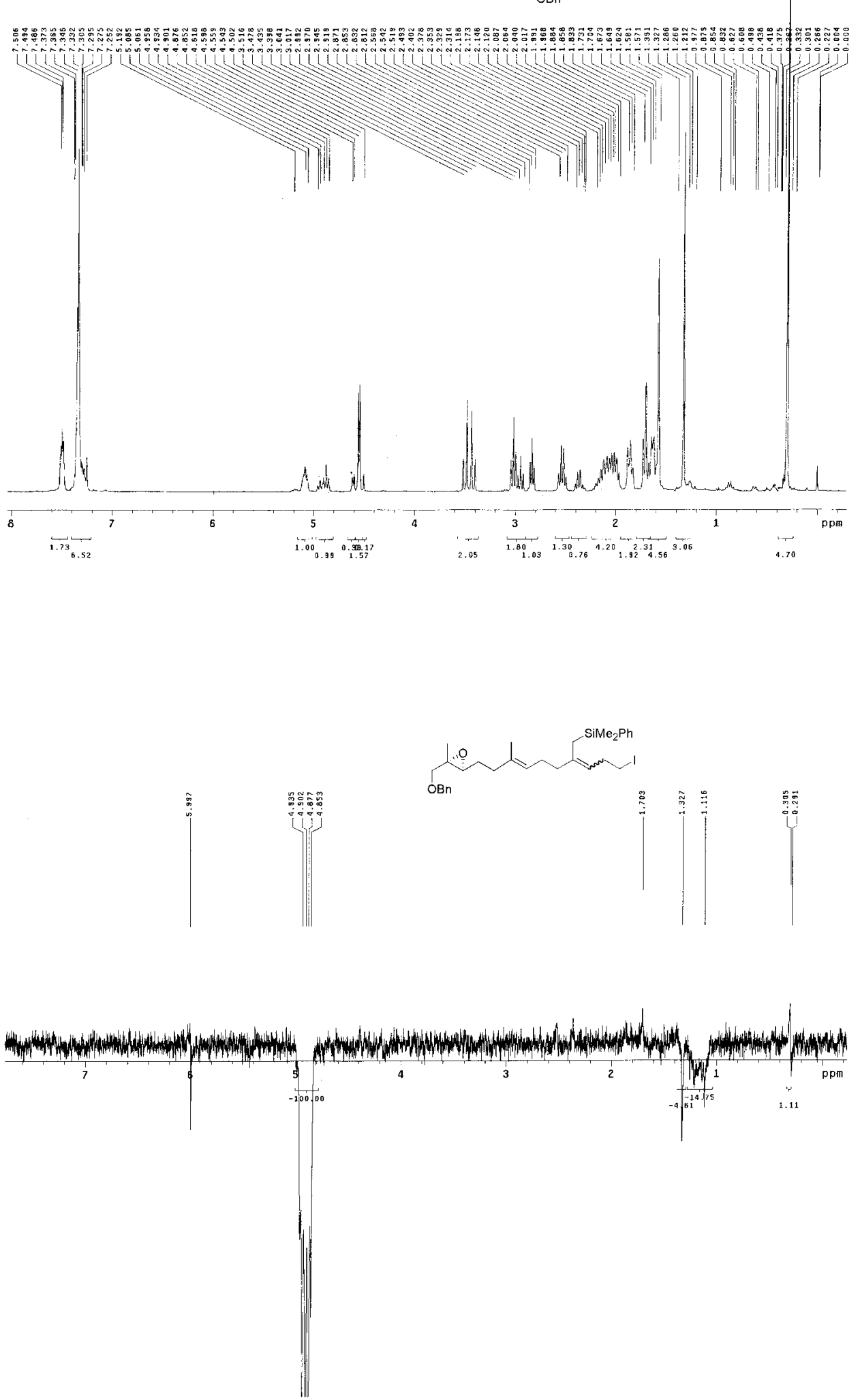


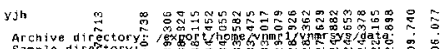

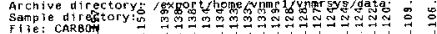

pulse sequenfe: sapul L
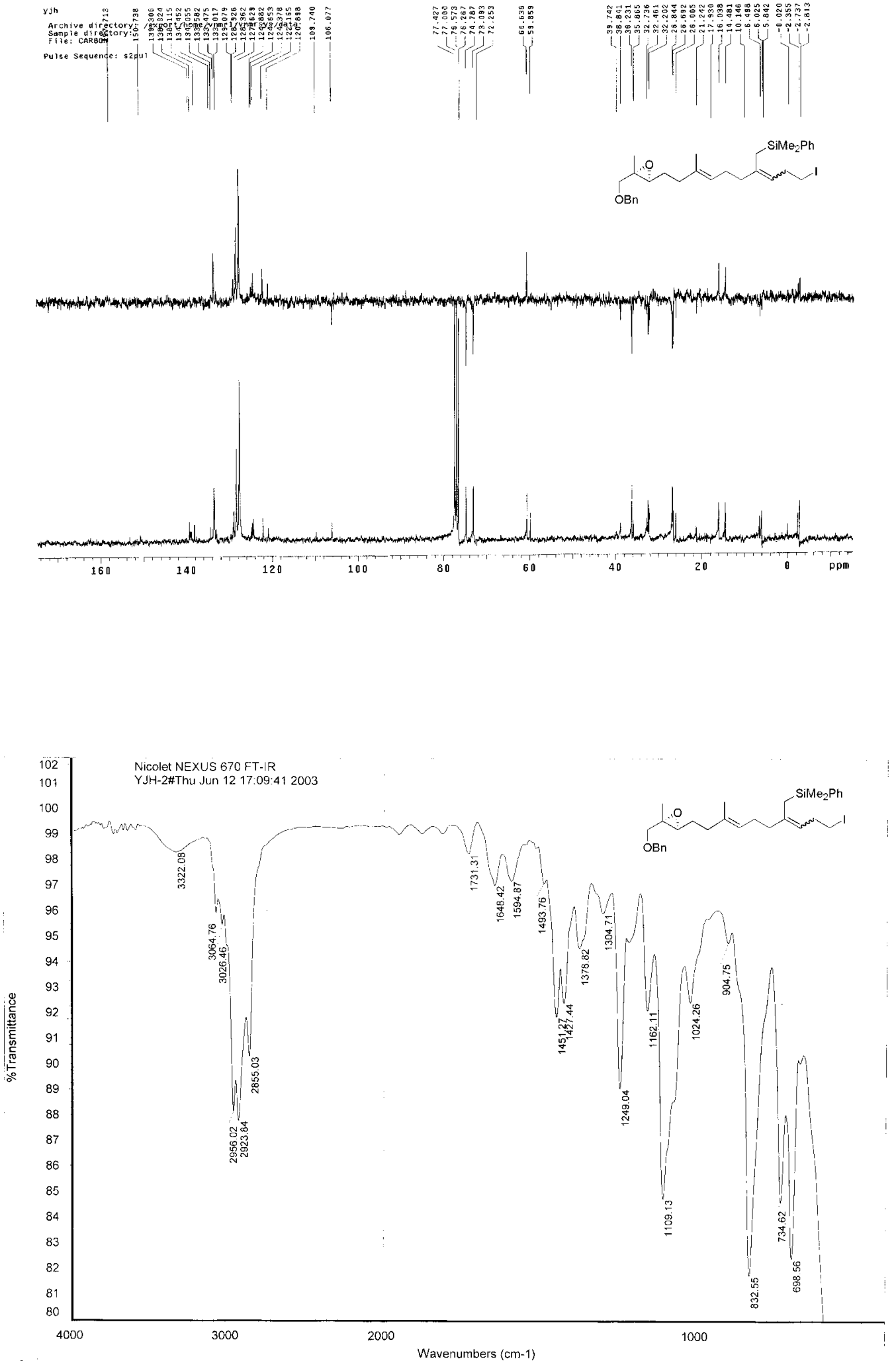
y)

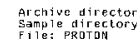

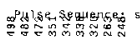

ininivini

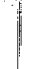
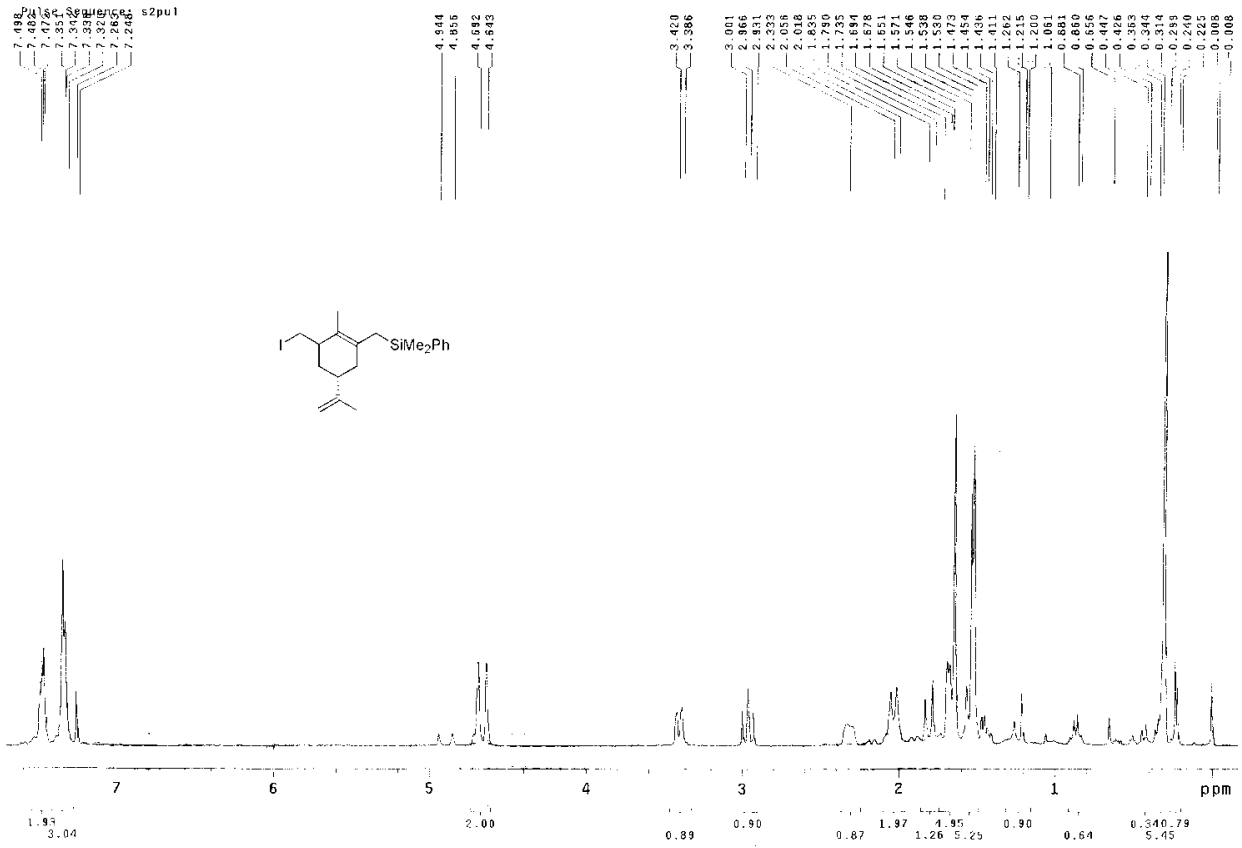

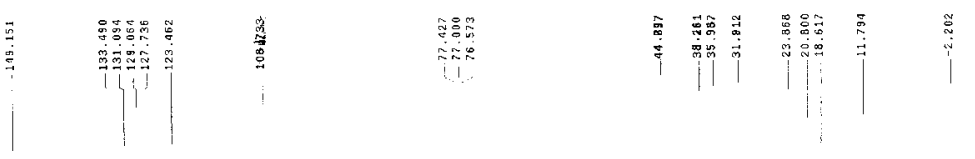

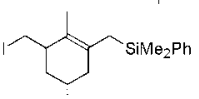

$\lambda$

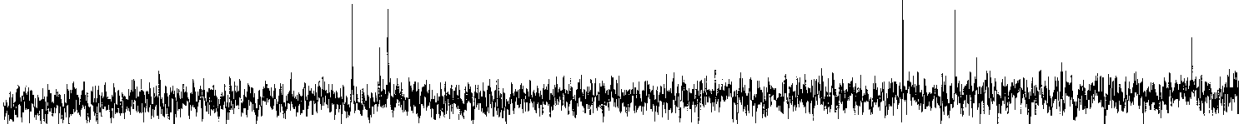

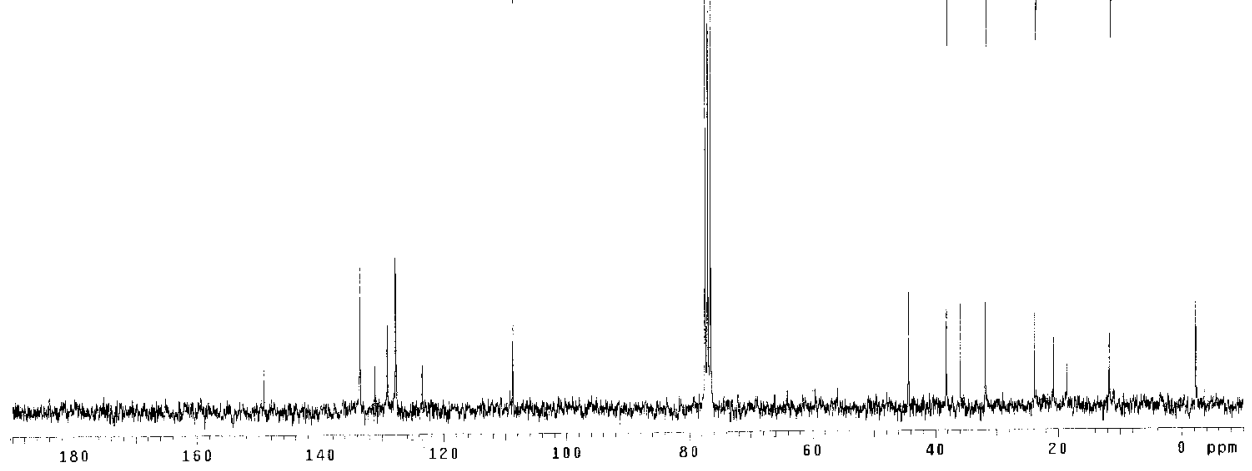




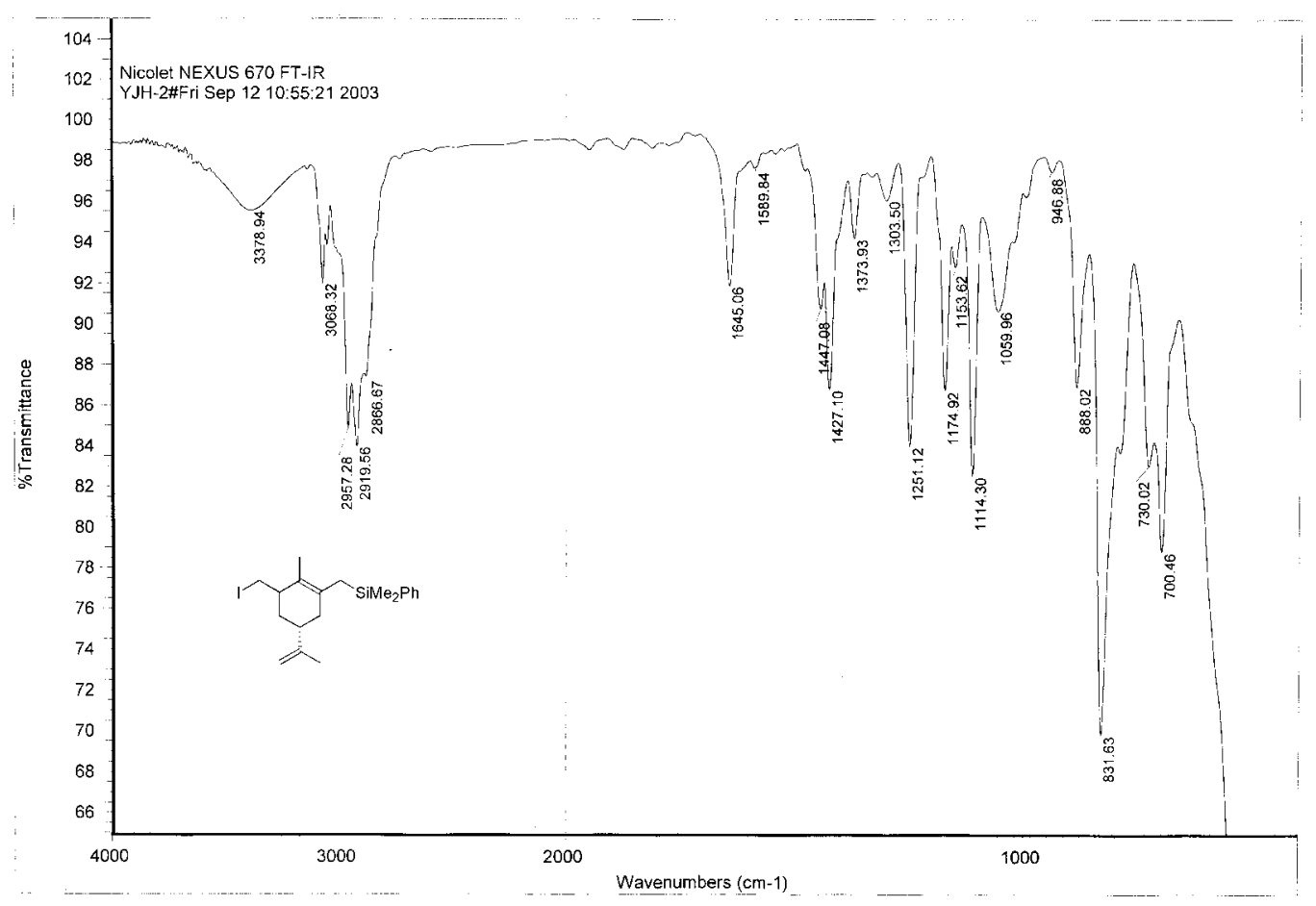

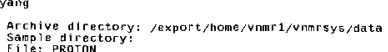

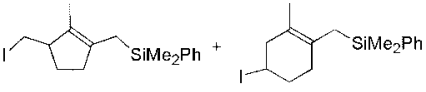

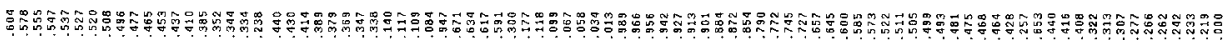

烈
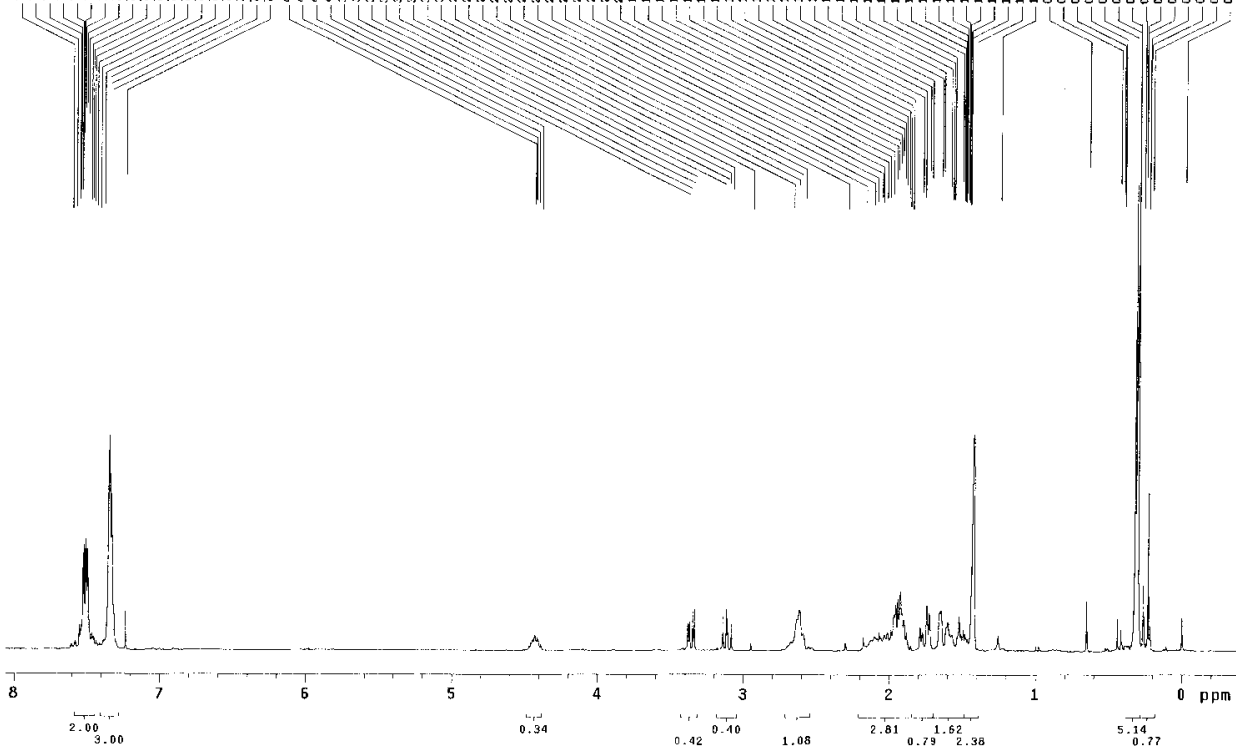

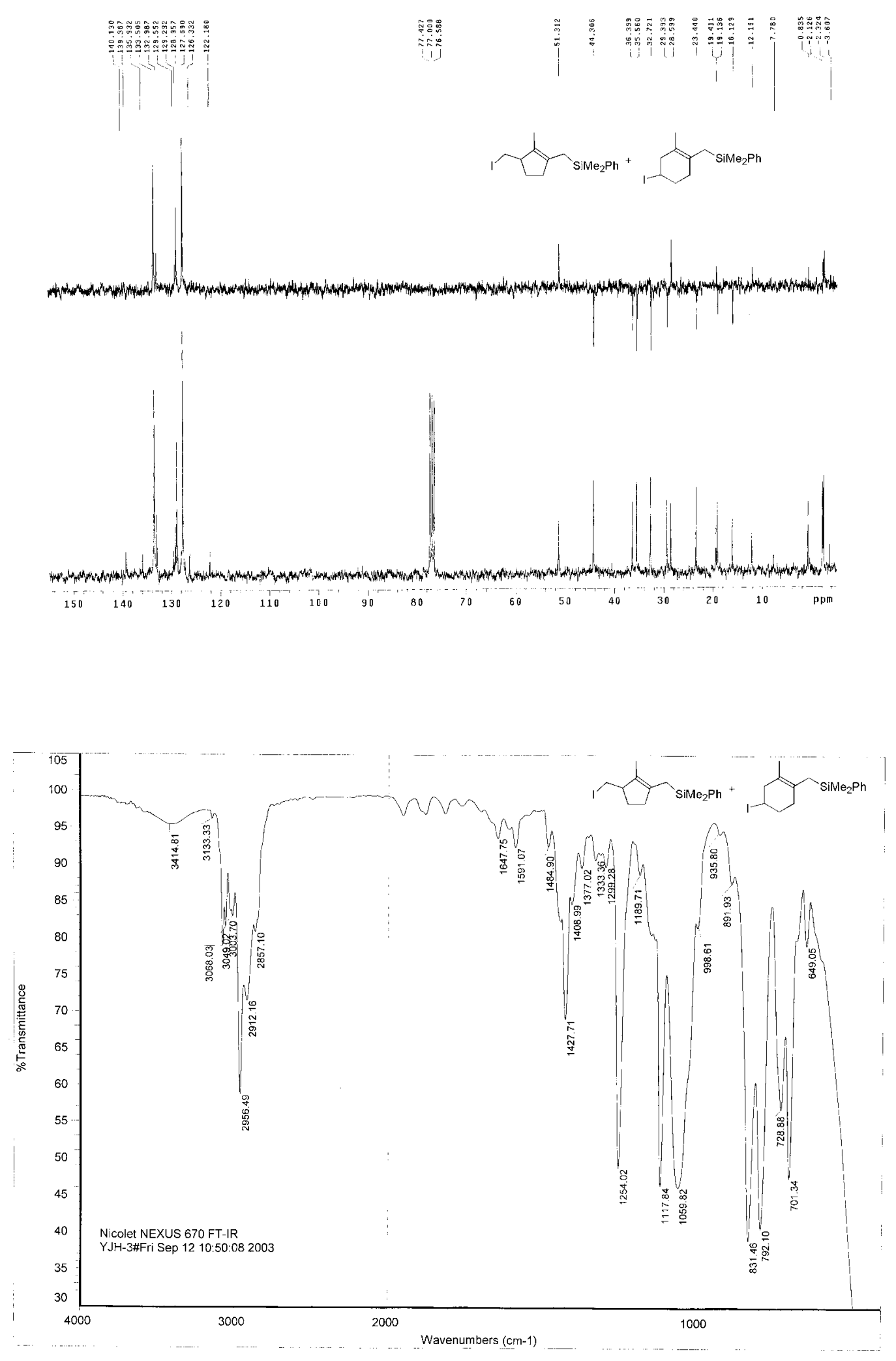
wher
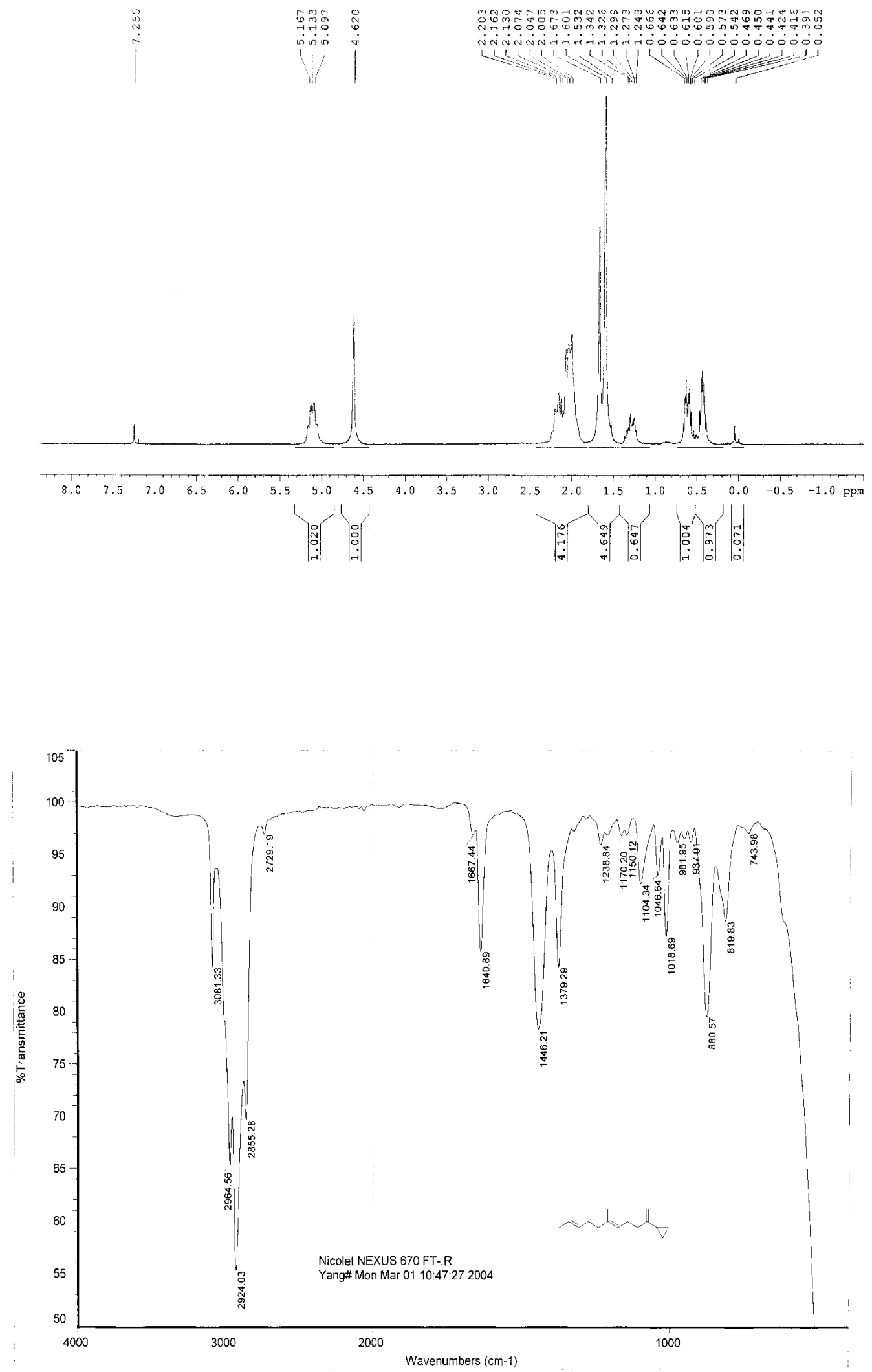

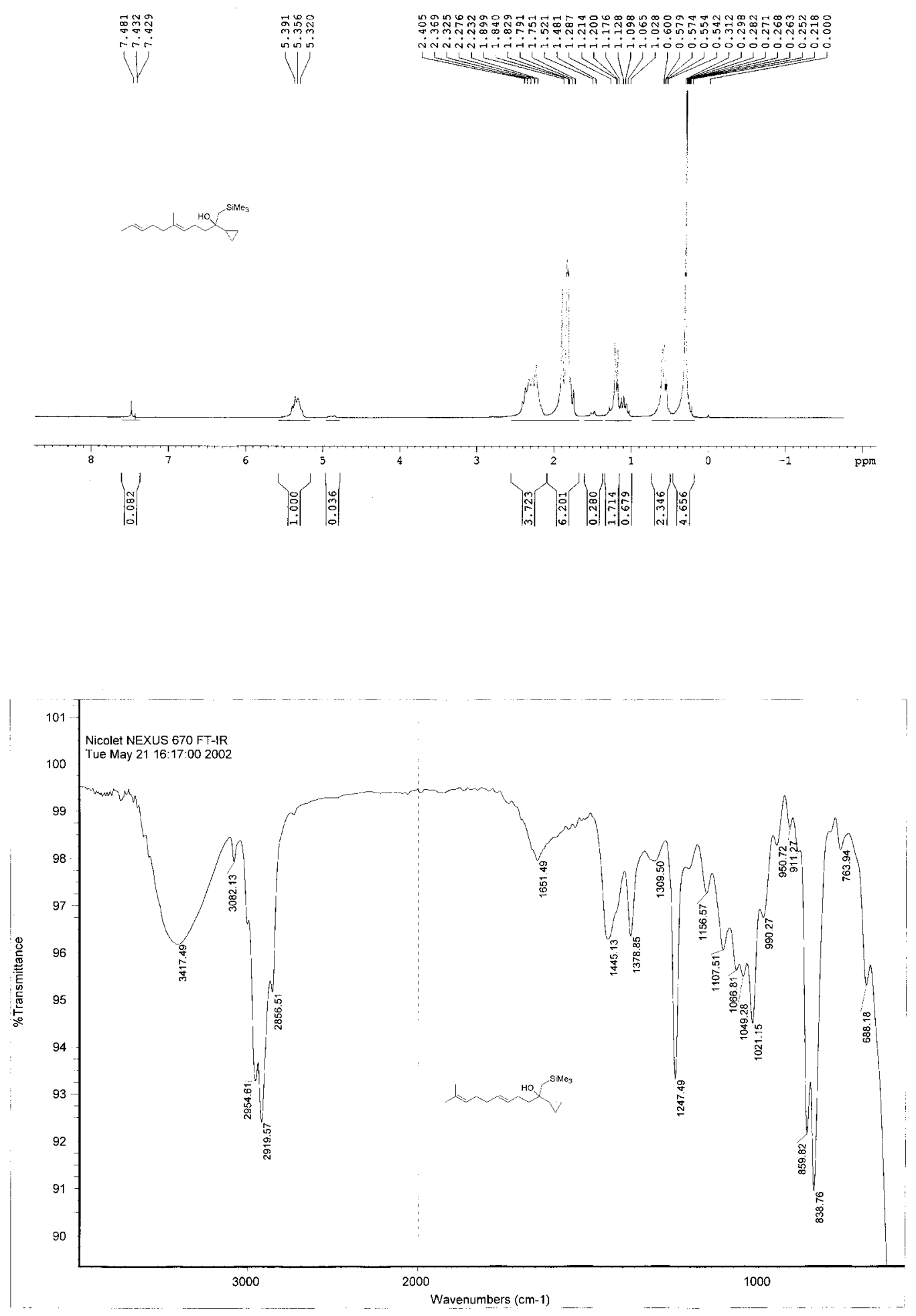

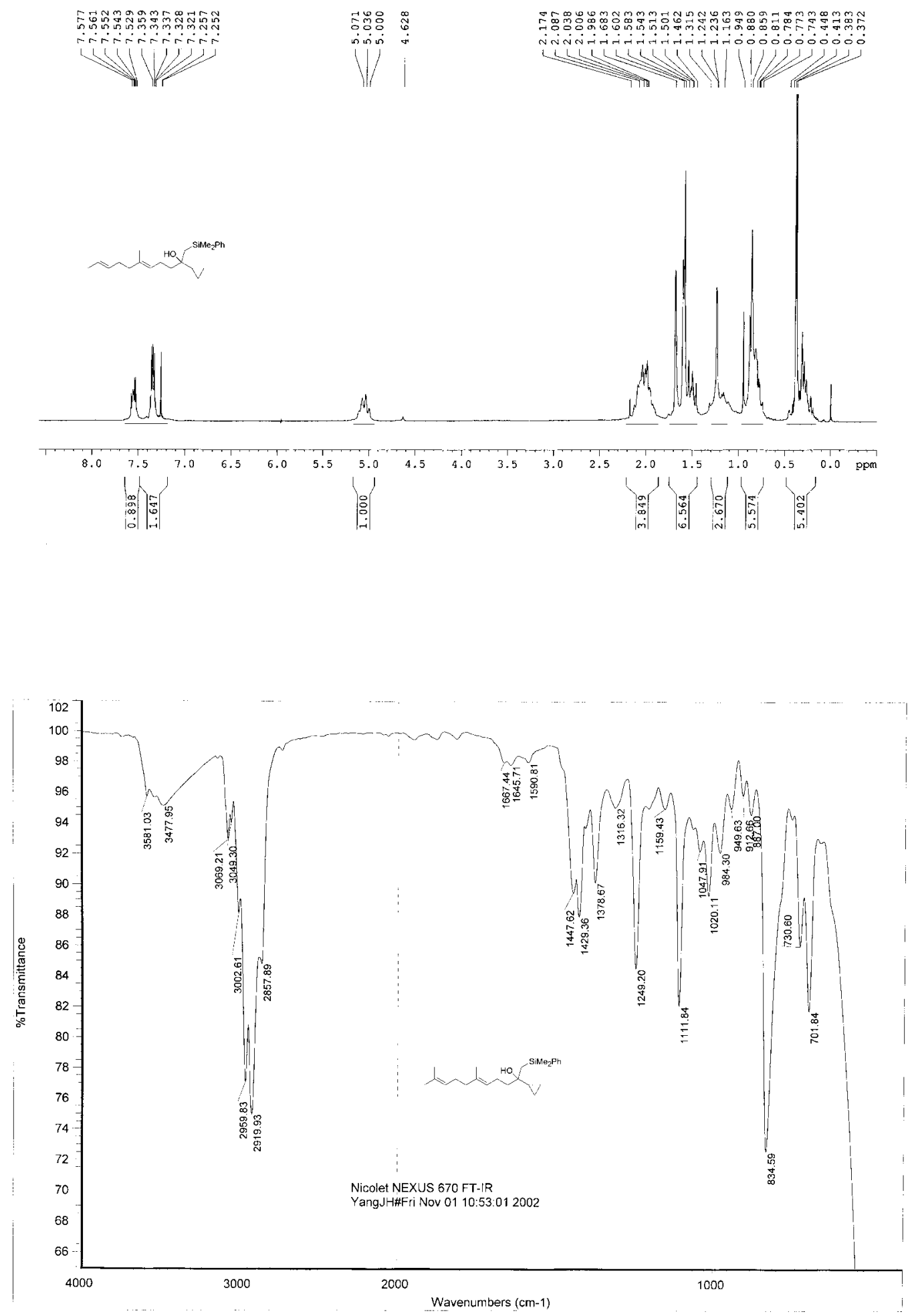
A8_2

Archive directory:
Samplexport/home/liwd/unmesys/data
Filectory:

Pulse sequence: s2pul

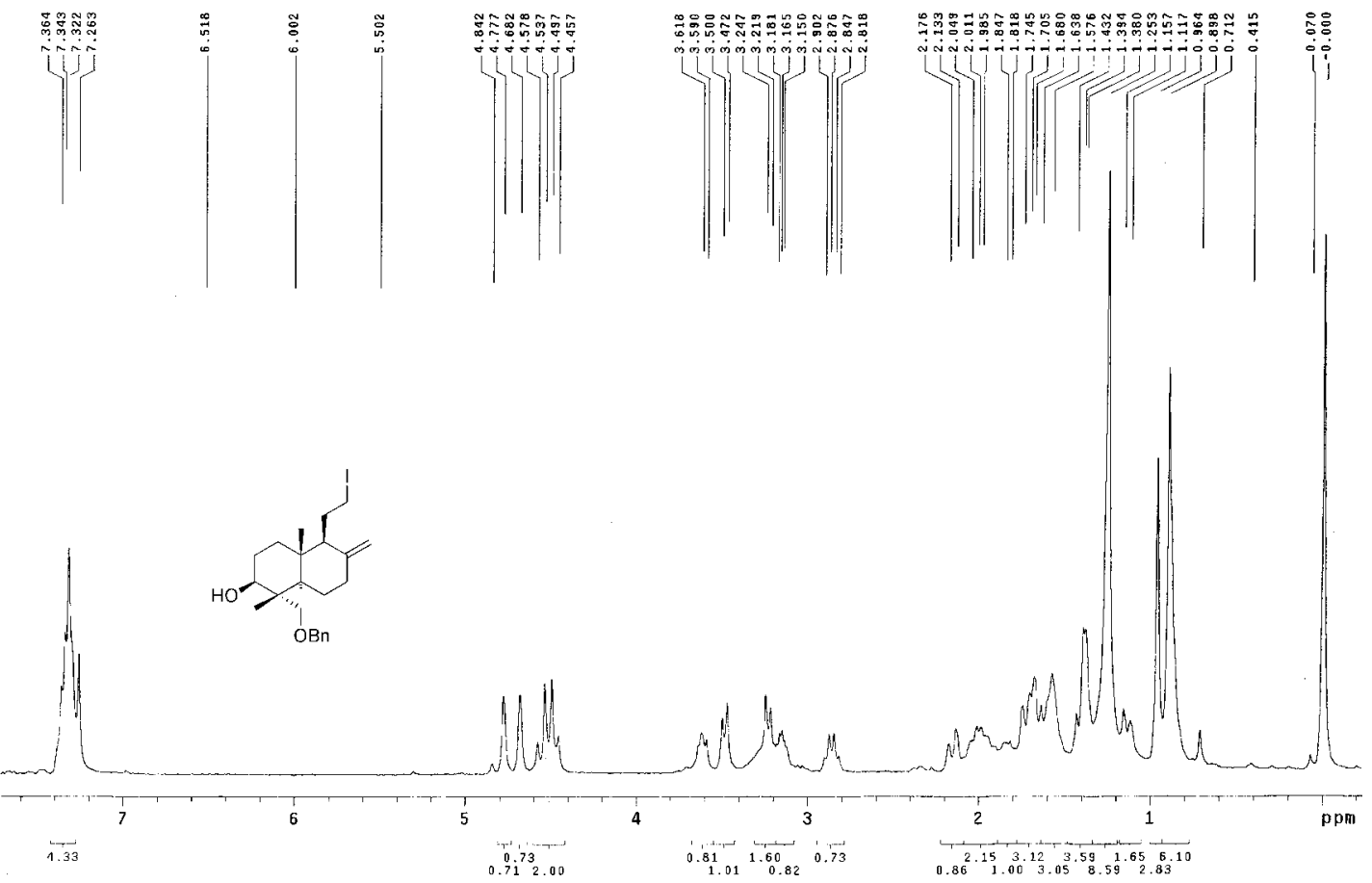

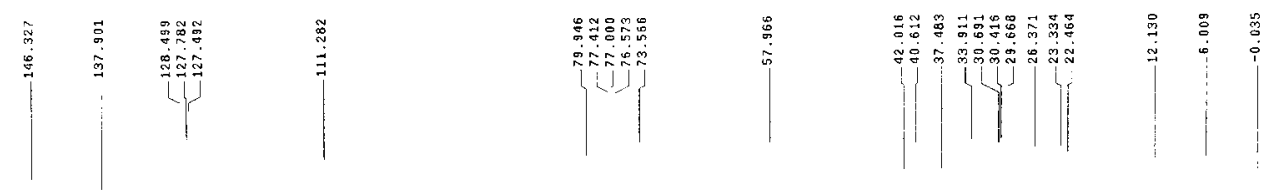
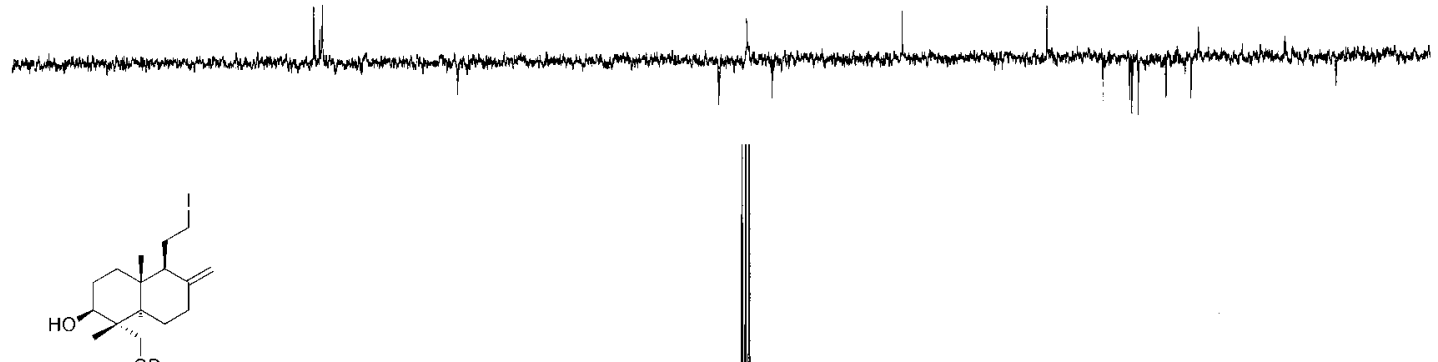

Bn

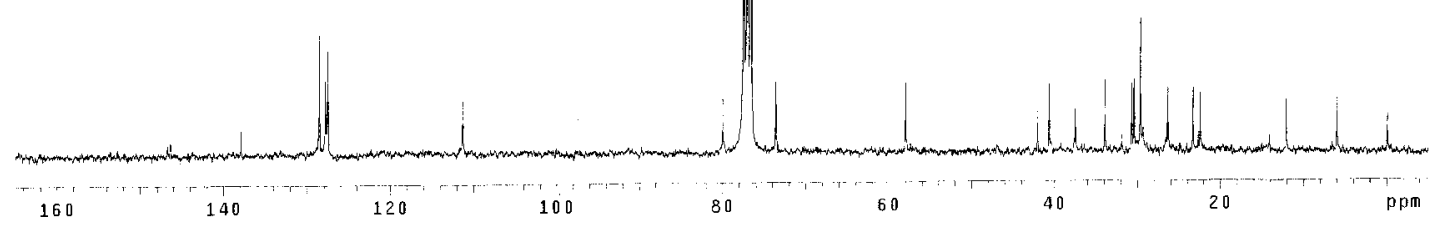




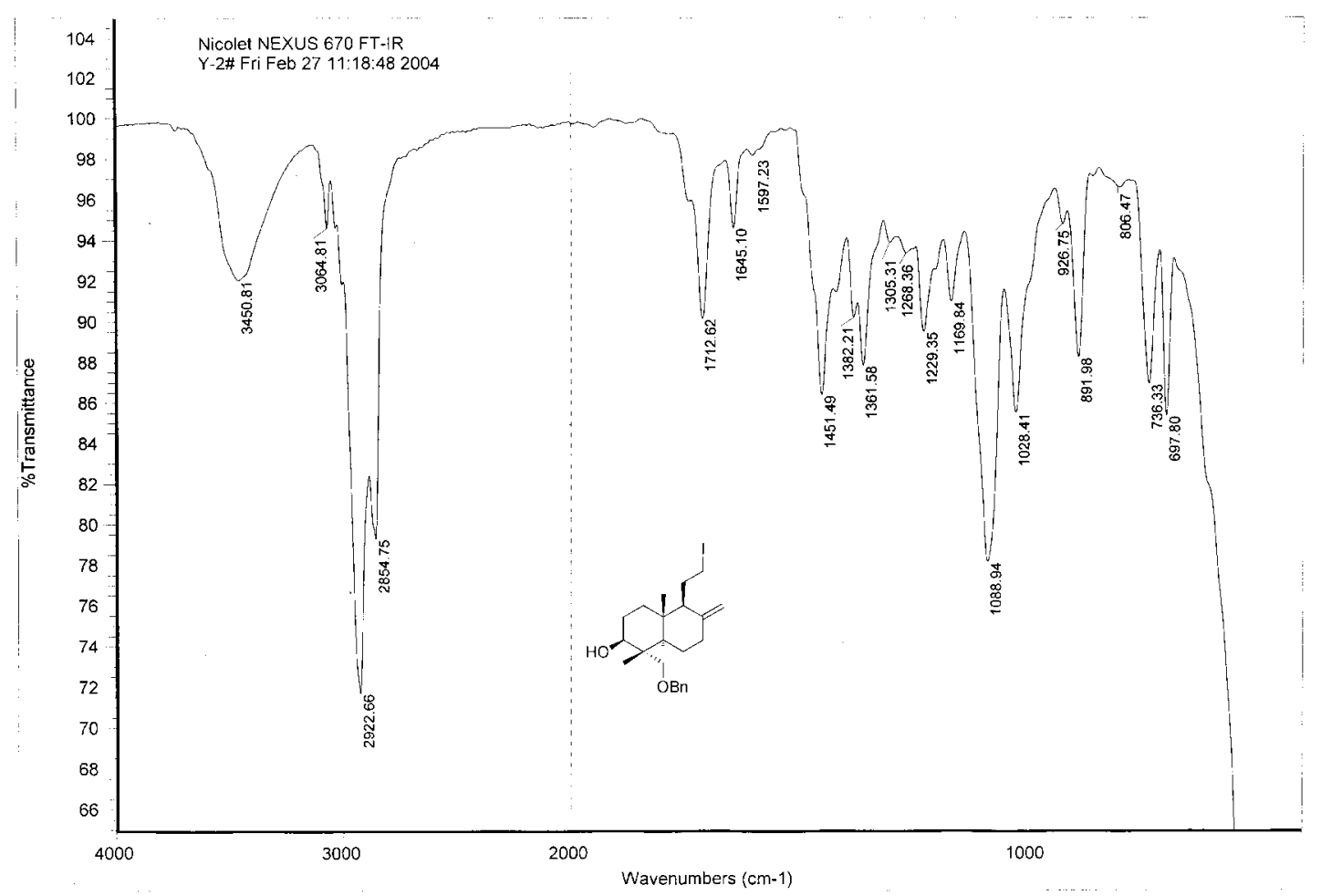

at. 1

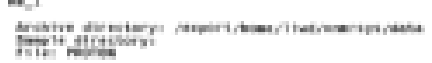

mus newases atmon
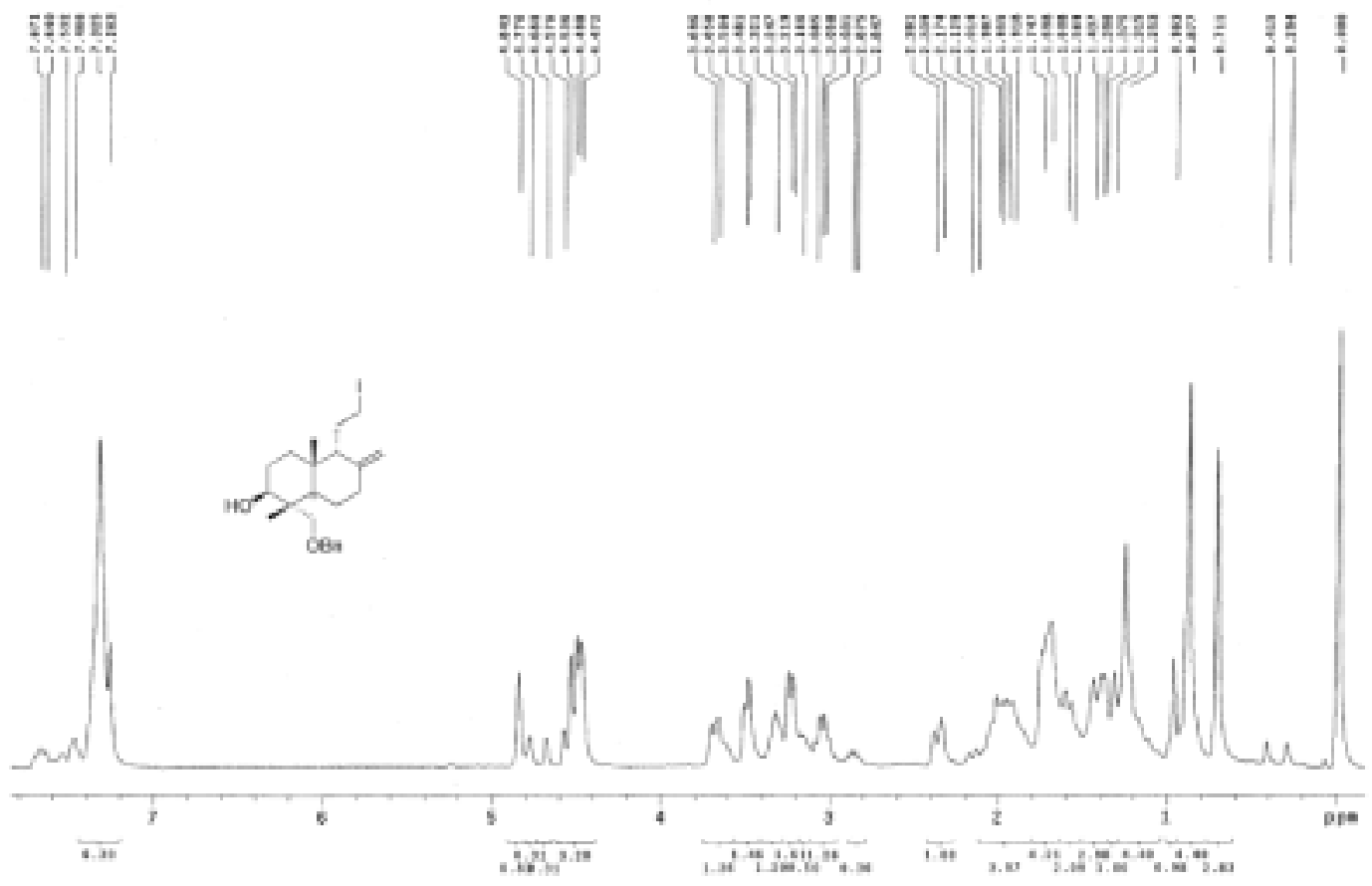


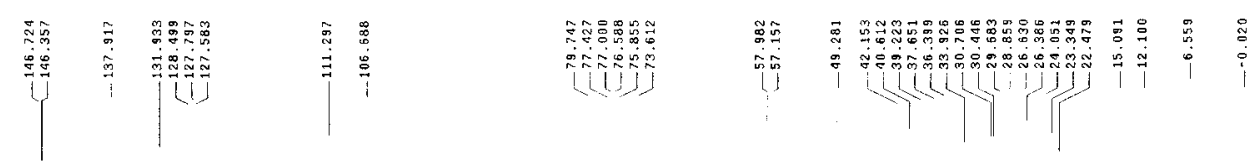
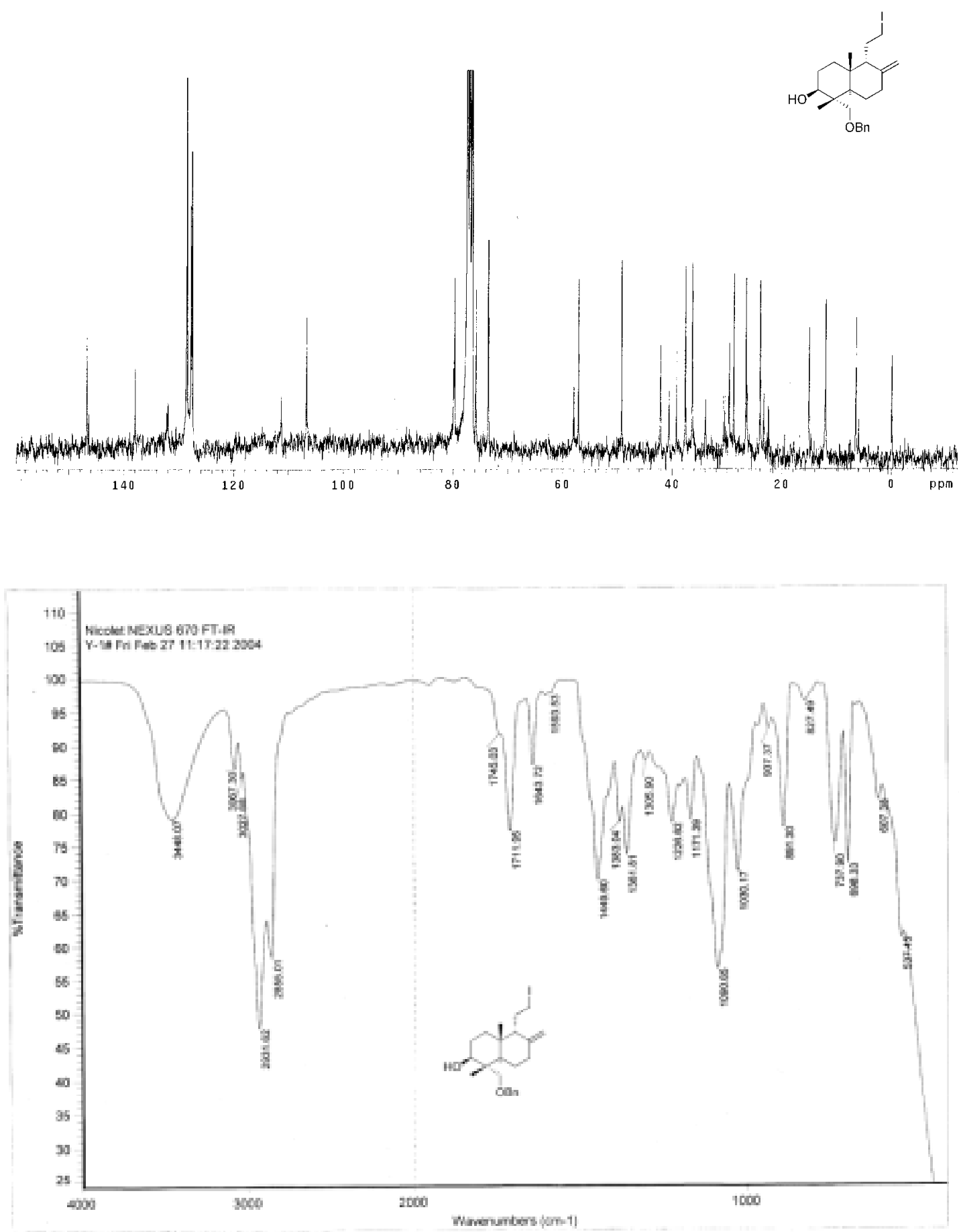
File : C: \HPCHEM $\backslash \backslash$ DATA $\backslash 30109 . D$

Operator : HP5988A MS, Lanzhou University

Acquired : 1 Mar 2004 9:49 using AcclMethod DIP700

Sample Name: Yang Jing-hui, 468

Misc Info : State Key Lab of Applied Organic Chemistry

Vial Number: 1

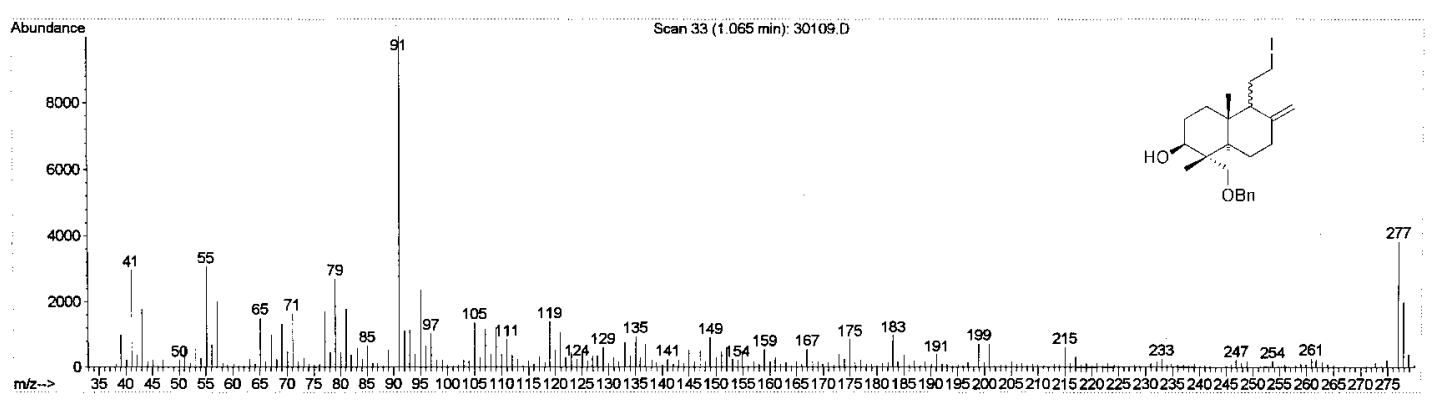

Abundance

$\operatorname{scan} 33(1.065 \mathrm{~min}): 30109 . \mathrm{D}$
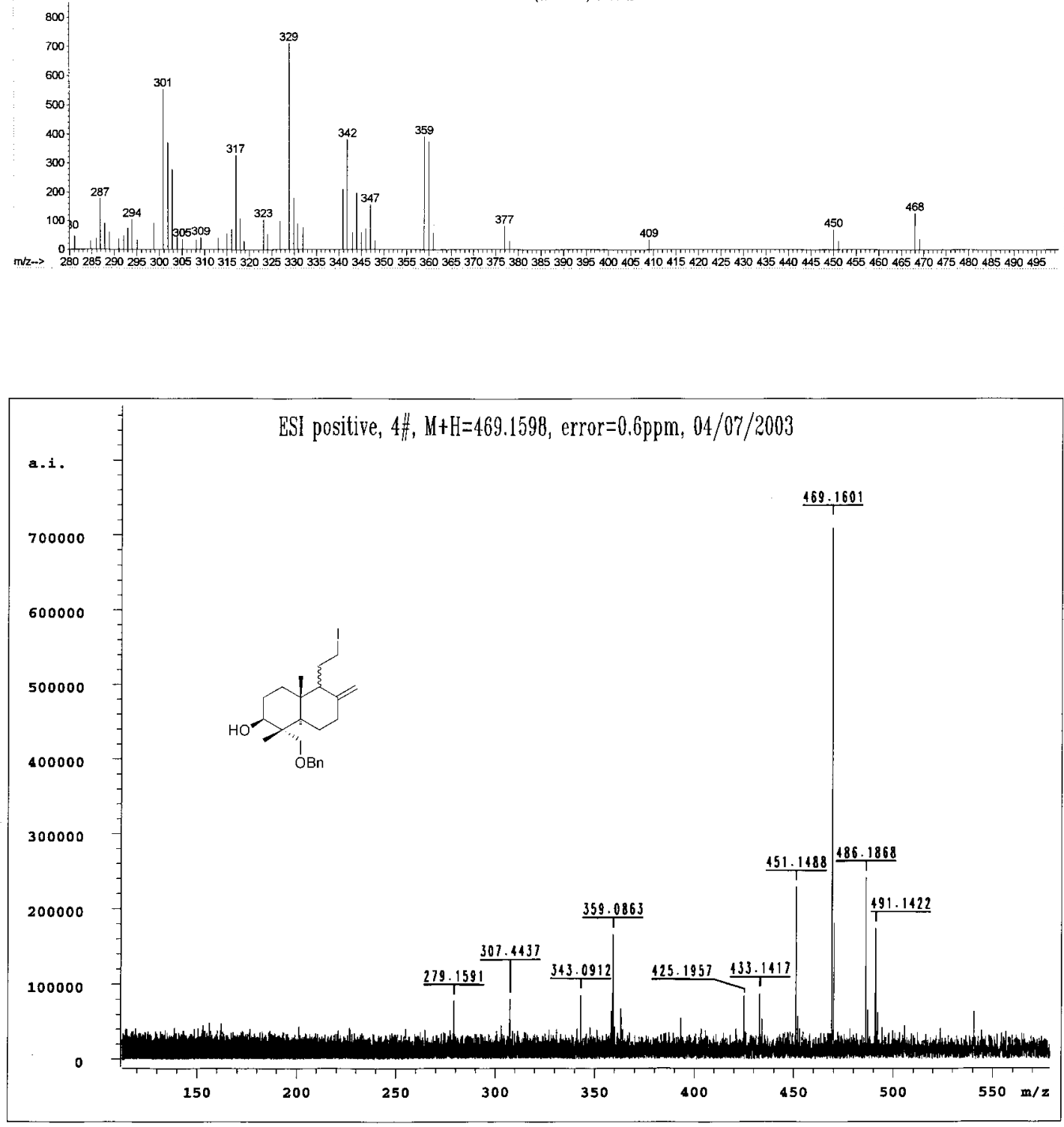

/u/data/TRAINING/Yangjinhui0704/4/pdata/1 xspec Fri Jul 4 10:55:33 2003 